\title{
Cardiomyocyte GATA4 functions as a stress- responsive regulator of angiogenesis in the murine heart
}

\author{
Joerg Heineke,, ${ }^{1}$ Mannix Auger-Messier, ${ }^{1}$ Jian Xu, ${ }^{1}$ Toru Oka, ${ }^{1}$ Michelle A. Sargent, ${ }^{1}$ Allen York, \\ Raisa Klevitsky, ${ }^{1}$ Sachin Vaikunth, ${ }^{1}$ Stephen A. Duncan, ${ }^{2}$ Bruce J. Aronow, ${ }^{1}$ Jeffrey Robbins, ${ }^{1}$ \\ Timothy M. Cromblehol, ${ }^{1}$ and Jeffery D. Molkentin ${ }^{1}$ \\ 1Department of Pediatrics, Cincinnati Children's Hospital Medical Center, University of Cincinnati, Cincinnati, Ohio, USA. 'Department of Cell Biology, \\ Neurobiology and Anatomy, Medical College of Wisconsin, Milwaukee, Wisconsin, USA.
}

\begin{abstract}
The transcription factor GATA4 is a critical regulator of cardiac gene expression, modulating cardiomyocyte differentiation and adaptive responses of the adult heart. We report what we believe to be a novel function for GATA4 in murine cardiomyocytes as a nodal regulator of cardiac angiogenesis. Conditional overexpression of GATA4 within adult cardiomyocytes increased myocardial capillary and small conducting vessel densities and increased coronary flow reserve and perfusion-dependent cardiac contractility. Coculture of HUVECs with either GATA4-expressing cardiomyocytes or with myocytes expressing a dominant-negative form of GATA4 enhanced or reduced HUVEC tube formation, respectively. Expression of GATA4 in skeletal muscle by adenoviral gene transfer enhanced capillary densities and hindlimb perfusion following femoral artery ablation. Deletion of Gata4 specifically from cardiomyocytes reduced myocardial capillary density and prevented pressure overload-augmented angiogenesis in vivo. GATA4 induced the angiogenic factor VEGF-A, directly binding the Vegf-A promoter and enhancing transcription. GATA4-overexpressing mice showed increased levels of cardiac VEGF-A, while Gata4-deleted mice demonstrated decreased VEGF-A levels. The induction of HUVEC tube formation in GATA4-overexpressing cocultured myocytes was blocked with a VEGF receptor antagonist. Pressure overload-induced dysfunction in Gata4-deleted hearts was partially rescued by adenoviral gene delivery of VEGF and angiopoietin-1. To our knowledge, these results demonstrate what is to our knowledge a previously unrecognized function for GATA4 as a regulator of cardiac angiogenesis through a nonhypoxic, load, and/or disease-responsive mechanism.
\end{abstract}

\section{Introduction}

In the year 1990 cardiovascular disease surpassed infectious diseases as the leading cause of death worldwide, and by the year 2020 it is predicted to be the leading cause of all disability $(1,2)$. Coronary heart disease and atherosclerosis account for roughly two-thirds of all deaths from heart disease and hence have been deemed an epidemic $(3,4)$. Coronary heart disease is also the leading underlying cause of heart failure, which afflicts an estimated 5 million Americans, with approximately 400,000 new individuals diagnosed each year (4-6). Heart failure is defined as a progressive loss of ventricular pump function that produces inadequate tissue perfusion or reduced perfusion reserve and is characterized by key pathologic features such as ventricular dilation, additive loss of myocytes by apoptosis/necrosis, and rarefaction of capillaries $(7,8)$.

Coronary angiogenesis is induced in response to many diseasecausing stimuli that promote hypertrophy, in which it was suggested to play a protective role enabling continued functional compensation of the heart (9-11). For example, increased hemodynamic load in mice, sheep, and humans induces cardiac angiogenesis, although

Nonstandard abbreviations used: Ad-, adenovirus encoding; ChIP, chromatin IP; $\mathrm{CnA} \mathrm{Tg}$ mice, mice expressing the activated calcineurin cDNA; Dox, doxycycline; DTG, double transgenic; Gata4-Cre, cardiac-specific Gata4-deficient mice with a targeted allele and $\beta$-MHC-Cre-expressing transgene; HIF-1, hypoxia-inducible factor 1 ; $\mathrm{LDH}$, lactate dehydrogenase; $\alpha$-MHC, $\alpha$-myosin heavy chain; TAC, transverse aortic constriction; tTA, tetracycline transactivator.

Conflict of interest: The authors have declared that no conflict of interest exists. Citation for this article: J. Clin. Invest. 117:3198-3210 (2007). doi:10.1172/JCI32573. prolonged increases in afterload eventually leads to decompensation associated with reduced angiogenesis and cell death $(7,9,12-14)$. Important angiogenic cytokines such as VEGF-A and angiopoietin-1 are secreted by cardiomyocytes, from which they can directly signal to adjacent capillaries to enhance vessel and capillary formation $(15-18)$. Since angiogenesis is a complex process that involves the coordinated regulation of multiple gene products, it is not surprising that select transcription factors serve as master regulators of the entire program. During hypoxia the transcriptional regulator hypoxia inducible factor 1 (HIF-1) is activated through a specific hypoxia-sensing mechanism that facilitates induction of the angiogenic gene program (19). Studies in isolated cardiomyocytes have shown that stretch can also regulate VEGF-A release, suggesting a nonhypoxia mechanism for also inducing angiogenesis $(20,21)$.

The zinc finger-containing transcription factor GATA4 is highly expressed in cardiomyocytes throughout embryonic development, postnatal growth, and adulthood, during which it functions as a critical regulator of cardiac differentiation-specific gene expression (22). In addition to regulating basal gene expression in the heart, GATA4 has been implicated as a mechanical load-responsive transcriptional mediator that can be activated by increases in cardiac afterload, vasopressin infusion, or through direct stretching of the ventricles in the isolated rat heart $(23,24)$. At the molecular level, GATA4 is activated by phosphorylation through MAPKs, suggesting a link with upstream stress-responsive signaling pathways $(25$, 26). GATA4 is also negatively regulated by glycogen synthase kinase $3 \beta \alpha$-mediated (GSK3 $\beta$-mediated) phosphorylation, such that sig- 
A

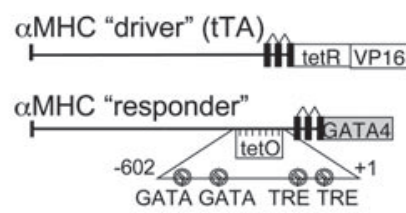

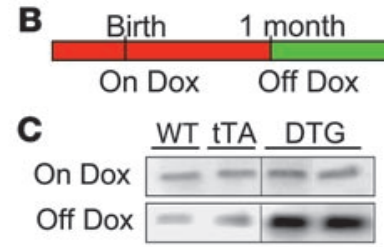

Line 20.8

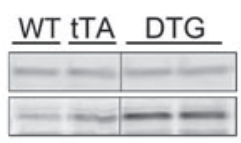

Line 21.2
D

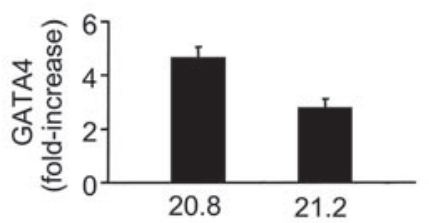

E
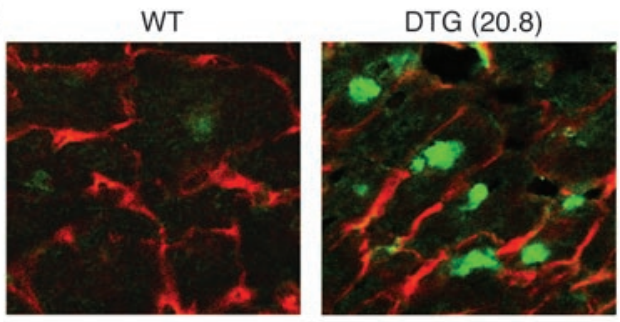

nal-dependent inactivation of GSK3 $\beta$ promotes greater GATA4 nuclear occupancy (27). Other evidence for GATA4 as an adaptive regulatory factor came from the simple observation that GATA4 overexpression by transgenesis in the mouse heart induced a mild form of hypertrophy after 6 months of age, although decompensation was not observed (28). Antithetically, we have recently shown that cardiomyocyte-specific deletion of Gata4 in mice reduces the hypertrophic response following pressure overload stimulation (29), collectively showing a prominent role for GATA4 in regulating myocyte growth. Deletion of Gata4 from the postnatal and adult heart also promoted cardiomyopathy with increasing age as well as acute functional decompensation following pressure overload in association with cell death (29). Partial Gata4 deficiency also enhanced stimulus-induced heart failure through a mechanism involving cardiomyocyte cell death and increased fibrosis (30).

Here we identify what we believe to be a novel function for GATA4 in the heart as a mediator of angiogenesis that facilitates compensation following injury. Loss of Gata4 by tissue-specific gene targeting in cardiomyocytes reduced capillary densities in the heart, while conditional overexpression of GATA4 using a tetracycline-regulated system significantly enhanced capillary densities. Mechanistically, GATA4 appears to induce angiogenic factors in cardiomyocytes, including direct regulation of the VEGF-A promoter. This paradigm of GATA4-dependent angiogenesis was also extended to a hind-limb ischemia model of injury, showing that GATA4 may serve as a global angiogenic regulator.

\section{Results}

Characterization of GATA4-inducible, cardiac-specific transgenic mice. To gain further insight into the function of GATA4 in the adult

\section{Figure 1}

Generation of cardiac-specific inducible GATA4 transgenic mice. (A) Schematic representation of the binary transgenic mouse system. (B) Time schedule of Dox treatment and transgene expression (red, no transgene expression; green, transgene expression). (C) Representative western blot analysis of GATA4 transgene expression at 1 month of age (mice still on Dox) and 3 months of age (mice off Dox for 2 months) in both transgenic mouse lines. The lanes were run on the same gel but were noncontiguous where indicated by the white line. (D) Quantification of GATA4 transgene expression in both mouse lines. (E) Immunofluorescence staining for GATA4 (green) counterstained with wheat germ agglutinin-TRITC (red) to outline cardiomyocytes. Original magnification, $\times 600$.

heart, we generated transgenic mice using an inducible system. A modified $\alpha$-myosin heavy chain $(\alpha-\mathrm{MHC})$ promoter was used as the responder transgene that produced cardiac-specific expression in the heart, regulated by doxycycline (Dox) administration/ withdrawal in the presence of a second $\alpha$-MHC promoter-driven tetracycline-controlled transactivator (tTA) transgene (Figure 1A) (31). Two GATA4 transgenic lines were extensively characterized and used throughout this study. Double transgenic (DTG) mice containing the driver and responder transgenes were maintained on Dox until 1 month of age, which prevented GATA4 transgene expression and any developmental effect (Figure 1, B and C). Upon Dox withdrawal, lines 20.8 and 21.2 showed 4.6 -fold and 2.5 -fold overexpression, respectively, of GATA4 in the heart at 3 months of age (Figure 1, C and D). Immunohistochemistry showed that GATA4 overexpression was specific to myocytes in the heart and that localization was nuclear (Figure 1E). Neither line showed any functional defect or alteration in cardiac performance as assessed by echocardiography in mice up to 7 months of age, indicating that overexpression of GATA4 at these levels was not pathological (see Table 1 and see below).

GATA4 enhances functional performance and capillary density in the heart. Enhanced expression of GATA4 in the heart caused a mild

\section{Table 1}

Baseline characteristics of inducible GATA4 transgenic mice

\begin{tabular}{|c|c|c|c|c|}
\hline & NTG & tTA & DTG (20.8) & DTG (21.2) \\
\hline \multicolumn{5}{|l|}{ Echocardiography } \\
\hline$n$ & 7 & 7 & 8 & 5 \\
\hline IVSth (mm) & $0.7 \pm 0.1$ & $0.7 \pm 0.1$ & $0.7 \pm 0.03$ & $0.8 \pm 0.1$ \\
\hline LVPWth (mm) & $0.7 \pm 0.1$ & $0.7 \pm 0.03$ & $0.6 \pm 0.03$ & $0.7 \pm 0.1$ \\
\hline $\operatorname{LVED}(\mathrm{mm})$ & $3.6 \pm 0.1$ & $3.9 \pm 0.1$ & $3.6 \pm 0.1$ & $3.6 \pm 0.2$ \\
\hline LVES (mm) & $2.1 \pm 0.1$ & $2.4 \pm 0.1$ & $2.2 \pm 0.1$ & $2.1 \pm 0.1$ \\
\hline FS $(\%)$ & $41.6 \pm 1.1$ & $38.3 \pm 1.5$ & $37.9 \pm 1.7$ & $42.8 \pm 0.7$ \\
\hline \multicolumn{5}{|l|}{ Gravimetry } \\
\hline$n$ & 10 & 11 & 8 & 5 \\
\hline HW/BW (mg/g) & $4.8 \pm 0.1$ & $4.9 \pm 0.1$ & $5.4 \pm 0.1^{A}$ & $4.8 \pm 0.2$ \\
\hline Lung/BW (mg/g) & $6.9 \pm 0.2$ & $6.3 \pm 0.2$ & $6 \pm 0.4$ & $5.3 \pm 0.4$ \\
\hline \multicolumn{5}{|c|}{$\begin{array}{l}\text { Mice were kept on DOX until } 1 \text { month of age. Analysis was carried out } \\
2 \text { months after DOX removal. AP }<0.05 \text { versus NTG, tTA, and DTG line } \\
21.2 \text { mice. NTG, nontransgenic; tTA, tTA transgenic ("driver") mice; } \\
\text { IVSth, end-diastolic intraventricular septal thickness; LVPWth, end- } \\
\text { diastolic left ventricular posterior wall thickness; LVED, left ventricular } \\
\text { end-diastolic diameter; LVES, left ventricular end-systolic diameter; FS, } \\
\text { fractional shortening; HW/BW, heart weight to body weight ratio; lung/ } \\
\text { BW, lung weight to body weight ratio. }\end{array}$} \\
\hline
\end{tabular}


A
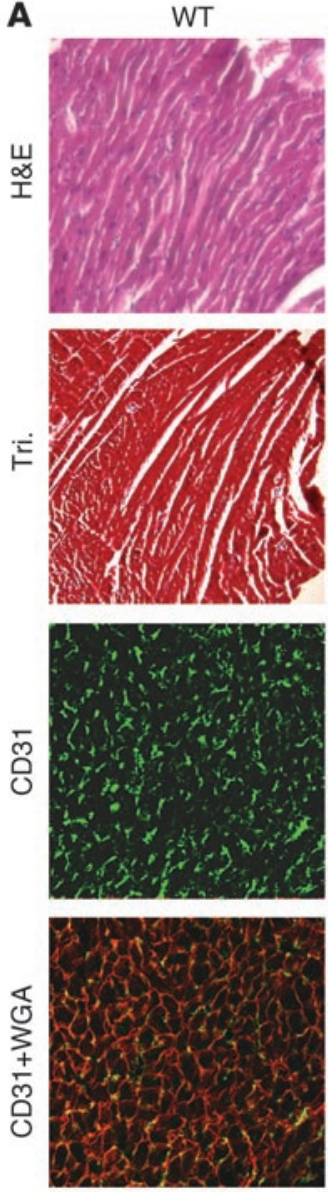

DTG (20.8)
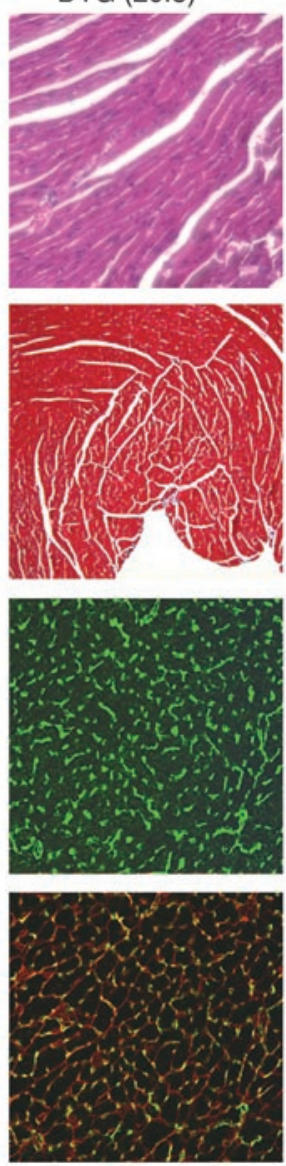

B

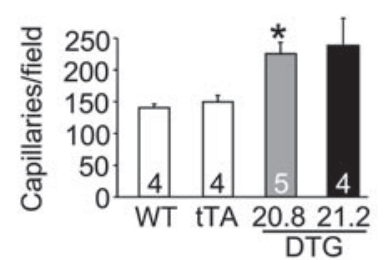

\section{D}
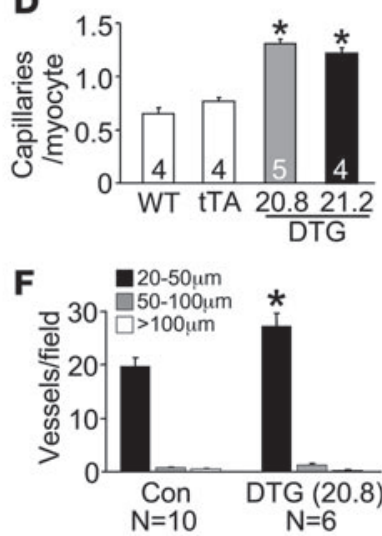

G

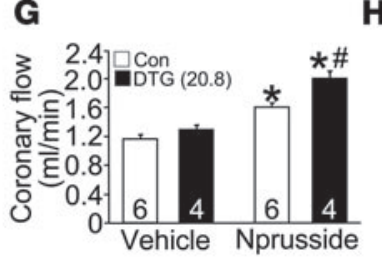

C

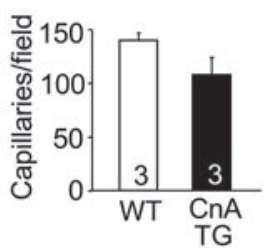

E

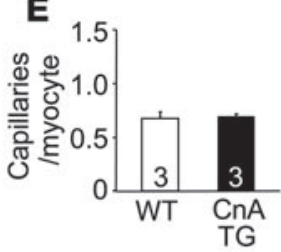

Figure 2

Induction of coronary angiogenesis by GATA4. (A) Representative staining of histological sections in WT and DTG mice by H\&E staining, Masson's Trichrome staining (Tri), CD31 (green), and CD31 + WGA (red). Original magnification, $\times 100$ (H\&E and Tri); $\times 400$ (CD31 and CD31 + WGA). (B-E) Quantification of absolute number of capillaries per microscopic field or capillaries per cardiomyocyte in control mice (Con; WT and tTA), DTG, and CnA Tg mice. ${ }^{*} P<0.01$ versus WT and tTA. (F) Conductance vessels quantified by size: small (20-50 um), medium (50-100 $\mu \mathrm{m})$, and large $(>100 \mu \mathrm{m}) .{ }^{*} P<0.01$ versus control (WT and tTA). (G) Coronary flow $(\mathrm{ml} / \mathrm{min})$ was measured in a working heart preparation in control (WT and tTA) and DTG mice with $(2 \mu \mathrm{g} / \mathrm{min})$ and without nitroprusside. ${ }^{*} P<0.01$ versus control vehicle; $\#<0.01$ versus control nitroprusside. (H) Cardiac contractile function measured as $+\mathrm{d} P / \mathrm{d} t(\mathrm{mmHg} / \mathrm{s})$ in the presence $(2 \mu \mathrm{g} / \mathrm{min})$ or absence of nitroprusside in control and DTG mice. ${ }^{*} P<0.01$ versus control with nitroprusside and control and DTG without nitroprusside. Numbers inside the bars indicate the number of animals analyzed. Hearts were sectioned and multiple sections per heart were quantified.

degree of cardiac hypertrophy (10\%) at 3 months of age in line 20.8 (high GATA4 overexpression) but not in line 21.2 (Table 1). This mild hypertrophy response was not pathologic, as ventricular chamber dimensions were unaffected and cardiac performance was maintained (low GATA4 overexpression) (Table 1). Histological analysis of hearts from line 20.8 also showed no discernable pathology or fibrosis, as assessed by H\&E and Masson's trichrome staining (Figure 2A). However, assessment of capillary and endothelial cell content in the heart by CD31 immunohistochemistry revealed a significant increase in GATA4 transgenic hearts (Figure 2A). Quantification showed significantly more capillaries per highpowered field (Figure 2B) and per single cardiac myocyte (Figure 2D). Interestingly, increased cardiac capillary density occurred independent of hypertrophy, since it was also observed in line 21.2, which has no increase in cardiac mass or cellular hypertrophy. Moreover, induction of cardiac hypertrophy due to expression of activated calcineurin did not increase capillary density (Figure 2, C and E). Thus GATA4 overexpression enhanced capillary density in the heart, likely independent of the hypertrophic response.

Given the effect of GATA4 on capillaries, we also investigated whether cardiomyocyte GATA4 overexpression was able to augment the number of larger conductance vessels. Vessels were identified by smooth muscle $\alpha$-actin immunostaining and parsed into different size ranges (Figure 2F). GATA4 activation in cardiac myocytes resulted in a significant increase in small conductance vessels (20-50 $\mu \mathrm{m}$ in diameter) but not larger vessels (Figure $2 \mathrm{~F}$ ). Importantly, this increase in small conductance vessels and capillaries was sufficient to significantly enhance the coronary reserve (total coronary flow under dilatory stimulation with nitroprusside) in DTG mice from line 20.8 when compared with the coronary reserve in control mice (Figure $2 \mathrm{G}$ ). Line 21.2, which expressed less GATA4 and showed no cardiac hypertrophy, also had a significantly higher coronary flow reserve than control mice (data not shown). Associated with this increase in flow capacity to the heart, 

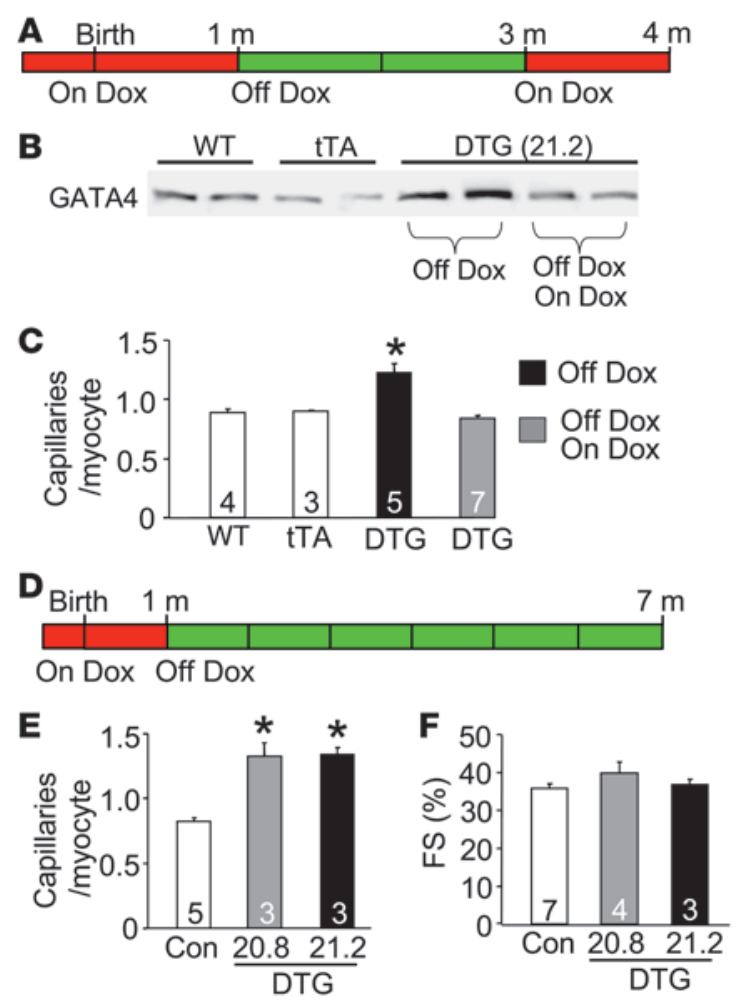

GATA4 DTG hearts showed an enhancement in cardiac contractility $(+d P / d t)$ compared with control hearts of the same strain using an ex vivo working heart preparation (Figure $2 \mathrm{H}$ ). These data indicate that enhanced GATA4 activity leads to an augmentation of myocardial angiogenesis, perfusion reserve, and perfusion-dependent cardiac contractility.

GATA4-regulated angiogenesis is reversible. The data presented above showed enhanced capillary and small conductance vessel densities from mice that were treated with Dox to prevent GATA4 transgene activation during development, then removed from Dox for 8 weeks to induce GATA4 expression in adulthood (Figure 1B). Here we conducted a similar experiment, but included another group that was placed back on Dox for 1 month to shut down the GATA 4 transgene between 3 and 4 months of age (Figure $3 \mathrm{~A}$ ). As expected, cardiac protein levels of GATA4 were higher in the induced mice at 4 months of age (separate control group), but overexpression was extinguished at 4 months of age in the group of mice to whom Dox was readministered for 1 month (Figure 3B). Associated with these changes in GATA4 protein levels, capillary densities were higher in the transgene-induced state and reduced to control levels when the transgene was shut off, indicating that a constant elevation of GATA4 is needed to maintain the augmented angiogenic response (Figure 3C).

To determine whether the effects of GATA4 overexpression were sustainable in the long term, we also analyzed a cohort of mice maintained in the induced state for 6 months (Figure 3D). Remarkably, the increase in capillary density in both GATA4 lines was still evident at 7 month of age (Figure 3E). This long-term increase in GATA4 expression and enhancement of capillary density was not pathologic, as fractional shortening remained optimal (Figure 3F).

Cardiac-specific deletion of Gata4 reduces capillary density. The potential physiologic relevance of our observations in GATA4-over-

\section{Figure 3}

Microvessel growth is strictly coupled to GATA4 expression and can be maintained for extended periods of time. (A) Time line of Dox treatment and GATA4 transgene expression (red, no transgene expression; green, transgene expression). DTG mice were taken off Dox at 1 month of age and then put back on Dox for 1 month at 3 months of age (Off Dox/On Dox group) or left off Dox (Off Dox group). (B) Representative western blot analysis of GATA4 protein expression in WT, tTA, and DTG Off Dox and Off Dox/On Dox mice. (C) Number of capillaries per cardiomyocyte in WT, tTA, and DTG Off Dox and Off Dox/On Dox mice. ${ }^{*} P<0.001$ versus WT, tTA, and DTG Off Dox/On Dox. (D) Time line of Dox treatment and GATA4 transgene expression for long-term analysis of GATA4 expression. (E) Number of capillaries per cardiomyocyte in control mice and DTG mice of lines 20.8 and 21.2 off Dox for 6 months. ${ }^{*} P<0.001$ versus control. (F) Fractional shortening (FS) determined by echocardiography in control and DTG mice of both lines 6 months off Dox. Numbers inside the bars indicate the number of animals analyzed. Hearts were sectioned and multiple sections per heart were quantified.

expressing transgenic mice is supported by the fact that endogenous GATA4 protein levels were normally increased in WT hearts following 2 weeks of pressure overload stimulation induced by transverse aortic constriction (TAC) (Figure 4A). This increase in GATA4 protein levels also corresponded with greater capillary densities in WT mice during the early compensatory hypertrophy phase (Figure 4B). However, extended periods (23 weeks) of pressure overload that typically result in decompensation no longer showed an increase in GATA4 protein or as much capillary density enhancement (Figure 4, A and B).

To further characterize the requirement of GATA4 in this process, we generated cardiac-specific Gata4-deficient mice using a Gata4-loxP targeted allele and a $\beta$-MHC-Cre-expressing transgene $\left(\right.$ Gata4$^{\beta}$-Cre $)$. We previously showed that the $\beta$-MHC-Cre transgene produces a $95 \%$ deletion of GATA4 protein from the adult heart in a myocyte-specific manner (29). Loss of Gata4 in these hearts produced a significant decrease in capillary densities at 2 and 6 months of age (Figure 4, C and D). Moreover, loss of Gata4 blocked the normal increase in cardiac capillary density that accompanies 1-2 weeks of pressure overload hypertrophy induced by TAC (Figure 4E). Physiologic hypertrophy induced by forced swimming exercise also enhanced capillary density in the myocardium of control mice but not in Gata4 ${ }^{\beta-C r e}$ mice (Figure 4F). The physiologic growth response is often associated with increased IGF-1 signaling in the heart, suggesting the possibility that GATA4 might respond to such signals in partially coordinating the angiogenic response. Indeed, IGF-1 treatment of cultured neonatal cardiomyocytes increased GATA4 phosphorylation at serine 105, an event that is known to augment GATA4 activity (Figure 4G). However, this increase in GATA4 serine 105 phosphorylation was blocked with dominant-negative MEK1, suggesting that IGF-1-mediated phosphorylation of GATA4 occurred through ERK1/2 (data not shown). Collectively, these results indicate that GATA4 is necessary for angiogenesis in response to physiologic hypertrophy and during the early compensatory phase of pathologic hypertrophy.

EF5 staining was used to evaluate whether the reduction in capillary density observed in Gata $4^{\beta-C r e}$ mice leads to myocardial hypoxia. EF5 staining, while qualitative, reliably indicates the relative degree of hypoxia in tissues (32). The specificity of the technique was demonstrated by strong EF5 staining from the infarcted myocardium and lack of staining in mice without EF5 administration (negative 


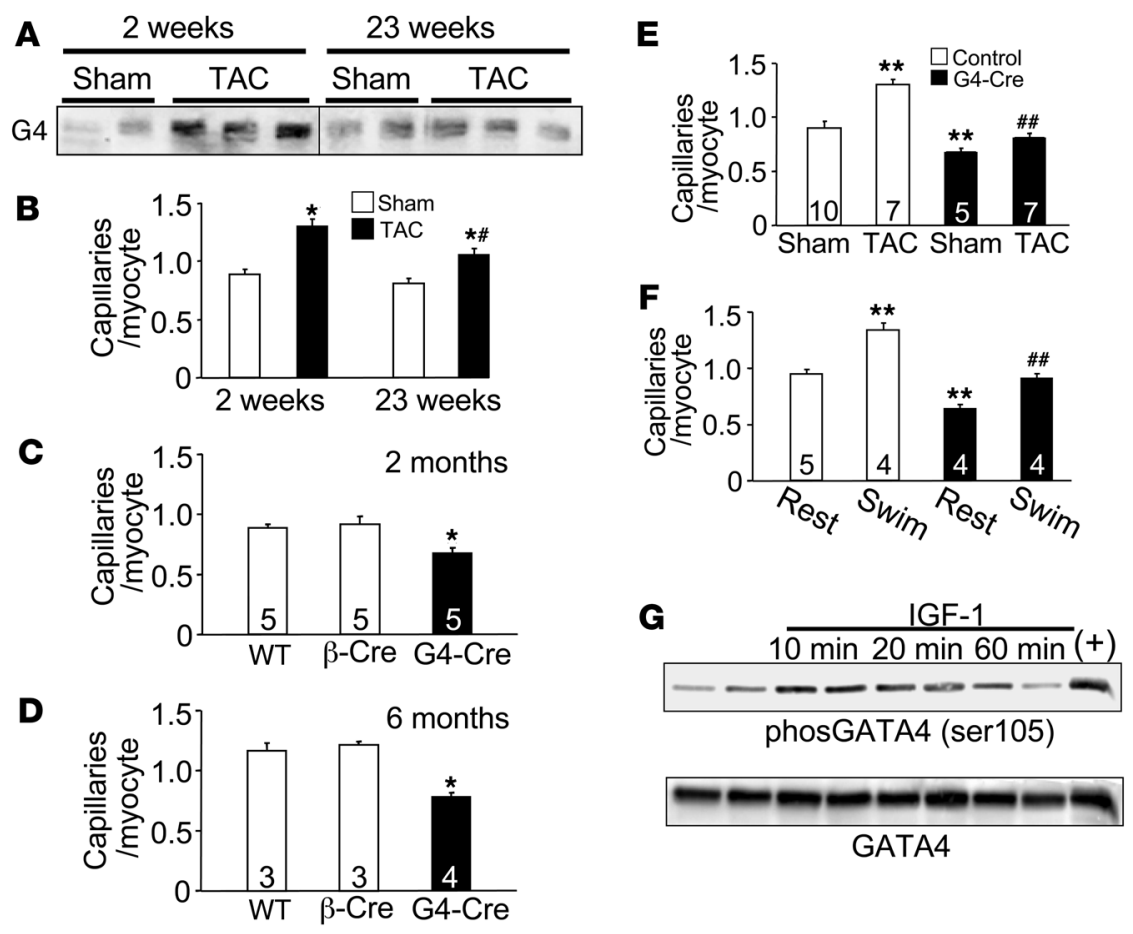

Figure 4

Cardiac-specific deletion of Gata4 compromises cardiac vessel content. (A) Western blot for GATA4 (G4) from hearts of WT mice subjected to sham operation or TAC for 2 or 23 weeks. The lanes were run on the same gel but were noncontiguous where indicated by the white line. (B) Capillary density in the hearts described in A. ${ }^{*} P<0.01$ versus sham; ${ }^{*} P<0.01$ versus TAC for 2 weeks. (C and D) Capillary density was assessed in hearts of WT, $\beta$-Cre transgenic, and Gata4 $\beta$-Cre (G4-Cre) mice at 2 months and 6 months of age. ${ }^{*} P<0.01$ versus WT and $\beta$-Cre. (E) Capillary density in control mice (WT and $\beta$-Cre) and Gata4 $\beta$-Cre mice 2 weeks after sham or TAC surgery. ${ }^{* *} P<0.001$ versus sham control; ${ }^{\# \#} P<0.001$ Gata4 $\beta$-Cre TAC versus control TAC. (F) Capillary density in control mice (WT and $\beta$-Cre) and Gata $4^{\beta}$-Cre mice after 3 weeks of swimming exercise and compared with cage-housed animals (rest). ${ }^{*} P<0.001$ versus control rest; \#\# $P<0.001$ versus control swim. (G) Western blot for total GATA4 or phospho-serine 105 GATA4 from neonatal cardiomyocytes infected with Ad-GATA4 24 hours prior to stimulation with IGF-1 (50 nM). The positive control (+) is from myocytes co-infected with Ad-MEK1. Numbers inside the bars indicate the number of animals analyzed. Hearts were sectioned and multiple sections per heart were quantified.

control) (Figure 5A). Control mice showed no detectable EF5 staining at baseline or after pressure overload, although Gata4 ${ }^{\beta-C r e}$ hearts showed noticeable, albeit low, staining at baseline and after TAC (Figure 5A). Consistent with this profile of hypoxia, hearts from Gata ${ }^{\beta}$-Cre mice showed a significant induction of HIF- $1 \alpha$ as well as the HIF-1 $\alpha$-responsive gene lactate dehydrogenase (LDH) in the heart after TAC (Figure 5B). However, LDH and HIF-1 $\alpha$ were not upregulated in Gata4 $\beta$-Cre mice at baseline, suggesting that pressure overload stimulation also contributed to gene induction.

Loss of vascular density directly predisposes Gata $4^{\beta}$-Cre mice to heart failure. We previously demonstrated that Gata4 ${ }^{\beta-C r e}$ mice develop cardiac dysfunction by 12 weeks of age that progressively worsens with time, ultimately resulting in death by the middle of adulthood (29). However, at 8 weeks of age Gata $4^{\beta-C r e}$ mice show no basal dysfunction, but upon TAC stimulation they rapidly decompensate and fail (29). Here we show that loss of capillary density significantly contributes to this phenotype of dysfunction and predisposition to heart failure in mice lacking cardiac Gata4. Eight-week-old con- trol mice (identical strain background and containing the $\beta$-MHC-Cre transgene) and Gata $4^{\beta-C r e}$ mice were first subjected to echocardiography, which showed no deficits in ventricular performance at this relatively young age (data not shown). Both groups of mice were then subjected to a cardiac-specific perfusion technique with either adenovirus encoding $\beta$-gal (Ad- $\beta$-gal) as a control or a combination of Ad-VEGF and Ad-angiopoietin-1 to increase vascular density in the heart as previously shown (9). Immediately following virus infusion, both groups of mice were subjected to a TAC surgical procedure and assessed 7 days afterward. We analyzed some hearts for the efficiency of gene transfer, using immunofluorescence against $\beta$-gal from uninfected and Ad- $\beta$-gal-infected mice. This showed widespread dispersion of the virus throughout the left and right ventricles and septum (Figure 5C and data not shown). Importantly, hearts collected after 7 days showed a significant increase in capillary density with Ad-VEGF plus Ad-angiopoietin- 1 in both control and Gata $4^{\beta}$-Cre mice, compared with Ad- $\beta$-gal infusion in control mice and Gata $4^{\beta-C r e}$ mice (Figure 5D). As previously shown by us, Gata4 ${ }^{\beta-C r e}$ mice subjected to TAC rapidly decompensate within 1 week, although Ad-VEGF plus Ad-angiopoietin-1 treatment significantly attenuated the degree of decompensation (Figure 5E). These results suggest that the increase in capillary density induced by Ad-VEGF plus Ad-angiopoietin-1 infusion partially protected Gata $4^{\beta-C r e}$ mice from pressure overload-induced dysfunction. These results further implicate vascular rarefaction as a key determinant of the cardiac pathogenesis in Gata4ß-Cre mice.

GATA4-overexpressing myocytes enhance angiogenesis in vitro. To begin to evaluate the cellular underpinnings of the GATA4-dependent angiogenic effect, we employed a Matrigel culture system using HUVECs. Primary neonatal cardiomyocytes were infected with Ad- $\beta$-gal (as a control), Ad-GATA4, or Ad-GATA4-engrailed (fusion protein) and 24 hours later replated on Matrigel, followed the next day by addition of HUVECs. We have previously shown that the GATA4-engrailed construct leads to potent inhibition of GATA4 transcriptional responses in myocytes (28). HUVECs form tube-like structures on Matrigel, as shown with the control Ad- $\beta$-gal-infected myocyte cocultures (Figure $6 \mathrm{~A}$ ). The quality and quantity of these tube-like structures was dramatically enhanced with Ad-GATA4-infected cardiomyocyte cocultures, while AdGATA4-engrailed produced fewer tube-like structures (Figure 6, A and B). Ad-GATA4-infected cocultures also enhanced HUVEC proliferation as assessed by BrdU incorporation, although GATA4engrailed infected cocultures had no effect on this index (Figure 6C). Thus GATA4 appears to program an angiogenic response within cardiomyocytes that can enhance endothelial cell activity through a paracrine mechanism. 
A
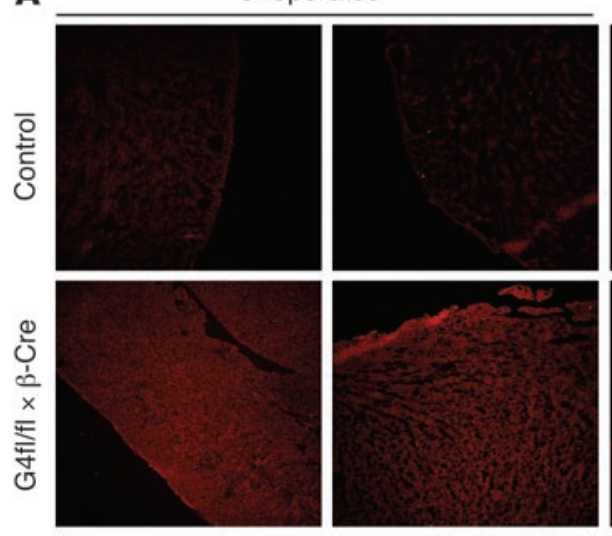

B
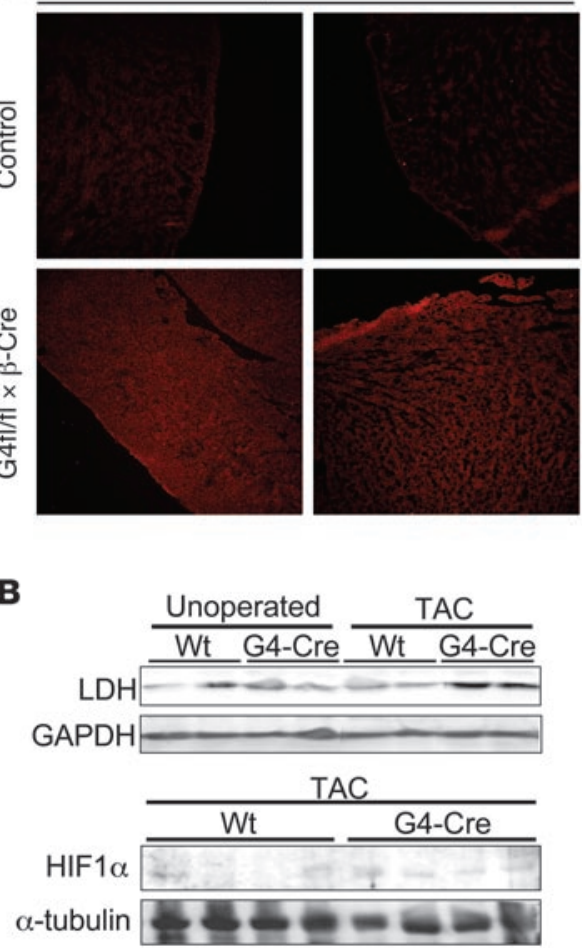

TAC
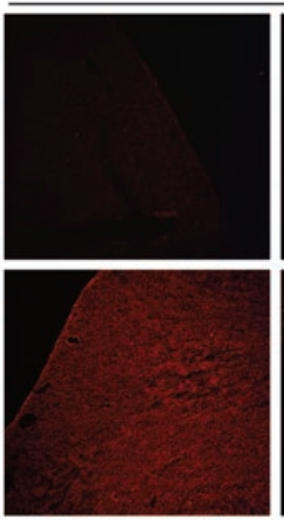

C

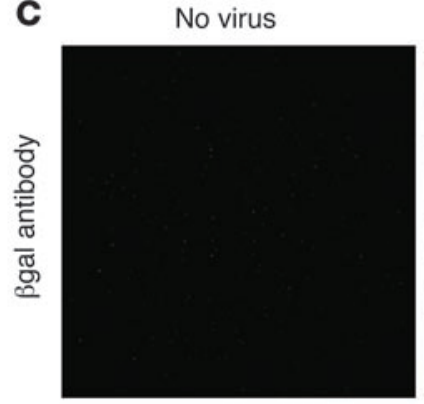

Neg con.
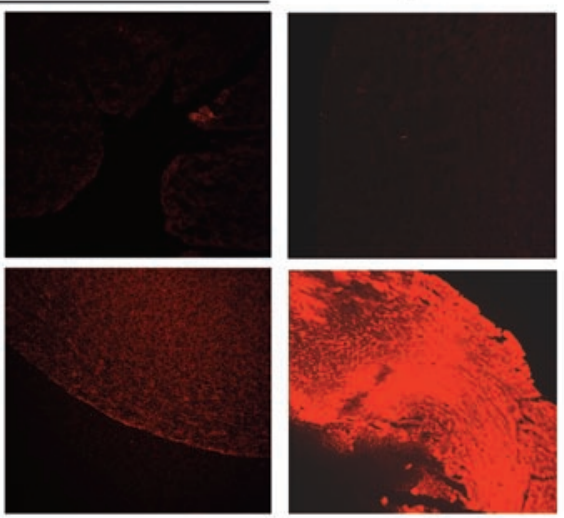

Ischemia

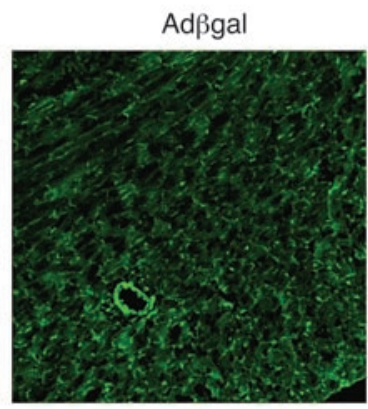

D
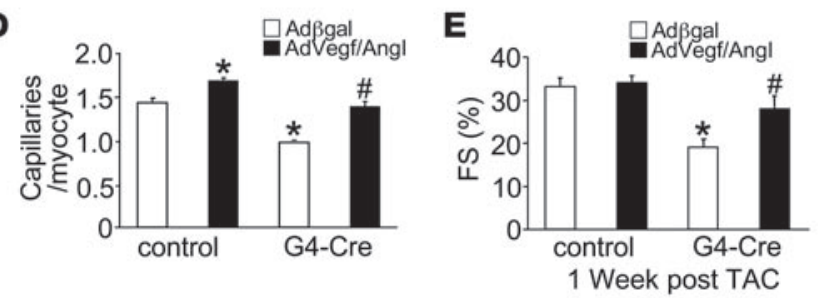

Figure 5

Assessment of the role of hypoxia and vascular rarefaction in promoting heart failure in heart-specific Gata4-deleted mice. (A) EF5 staining for the detection of ischemia from myocardial sections of WT mice and Gata4 ${ }^{\beta}$-Cre mice 2 weeks after TAC or without surgery as a control. Stronger intensity of red staining indicates ischemia. Mice with myocardial infarction were used as positive controls, and negative controls consisted of mice without EF5 injection. (B) Western blot analysis of myocardial HIF-1 $\alpha$ and LDH expression in WT and Gata4 $\beta$-Cre mice without surgery and 2 weeks after TAC. (C) Immunohistochemistry for $\beta$-gal protein expression (green) in cryopreserved histological sections from hearts previously treated with

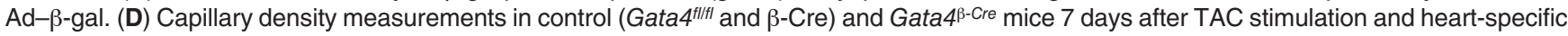
transduction with the indicated recombinant adenoviruses. ${ }^{*} P<0.01$ versus $\mathrm{Ad}-\beta$-gal control; $\# P<0.01$ versus $\beta$-gal treated Gata4 $\beta$-Cre mice. (E) Fractional shortening measured by echocardiography 1 week after TAC stimulation and transduction with the indicated viruses in control and Gata4 $\beta$-Cre mice. ${ }^{*} P<0.01$ versus Ad- $\beta$-gal control; ${ }^{*} P<0.01$ versus Ad- $\beta$-gal Gata4 $\beta$-Cre. Original magnification, $\times 100(\mathbf{A}) ; \times 200(\mathbf{C})$.

GATA4 induces a network of angiogenic factors, including VEGF-A. An Affymetrix array screen was performed in an attempt to define the molecular mechanisms whereby GATA4 might control angiogenesis and other homeostatic processes in the heart (Supplemental Table 1; supplemental material available online with this article; doi:10.1172/JCI32573DS1). A set of angiogenic genes was observed as significantly altered in the hearts of GATA4 DTG hearts compared with control hearts (Table 2). Select gene changes were detected with an RNase protection assay, including Vegfa, angiopoietin-1, CD31, and Tie2 (Figure 7A). CD31 and Tie2 are only expressed in endothelial cells, and their increased expression likely reflects the increased number of vessels that were present in the GATA4 DTG mice (33). Consistent with these observed changes in mRNA levels, hearts from GATA4 DTG mice showed greater VEGF-165 protein (a main product of the Vegfa gene), an effect that was also observed in isolated adult cardiomyocytes from DTG hearts, while expression of activated calcineurin in the heart had no effect (Figure 7B). In contrast, loss of GATA4 from the heart in Gata ${ }^{\beta-C r e}$ mice showed reduced VEGF-165 protein levels at baseline (Figure 7C) and even after the swimming exercise (Figure 7D). Acute overexpression of GATA4 in cultured cardiomyocytes also induced greater VEGF-165 protein accumulation in the cultured media as detected by western blotting (Figure 7E). Thus VEGF-A appears to be proximally regulated by GATA4 in the heart. Mechanistically, the induction of HUVEC tube formation on Matrigel by Ad-GATA4-overexpressing cocultured cardiomyocytes was 

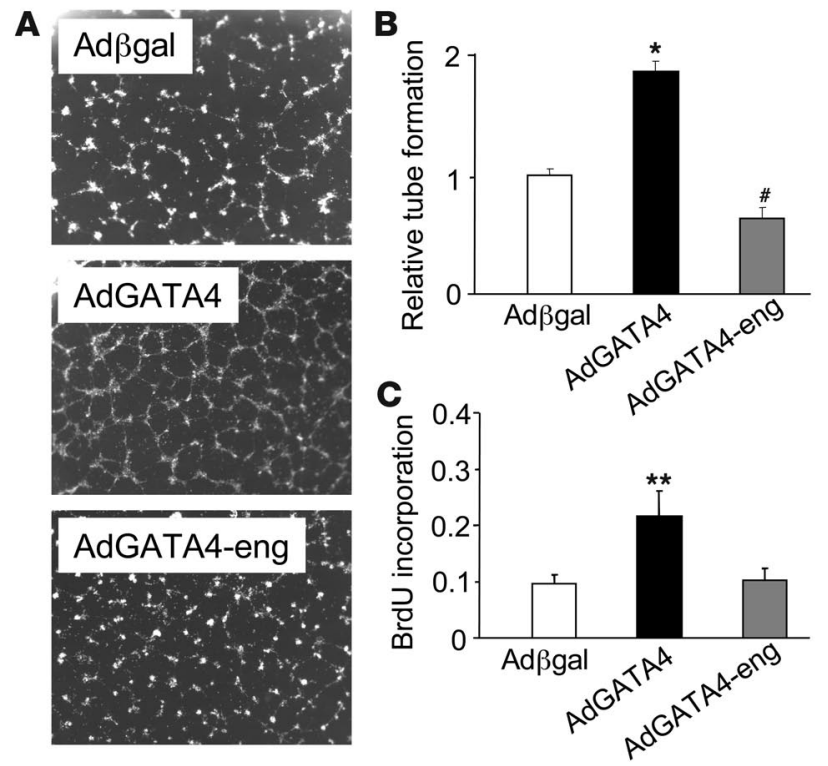

blocked with a VEGF receptor antagonist (CBO-P11), suggesting that GATA4 can enhance angiogenesis, in part through regulation of VEGF (Figure 7F). Collectively these results indicate that GATA4 directly or indirectly enhances angiogenesis, at least in part through VEGF gene expression.

To determine whether GATA4 can directly regulate VEGF expression we examined the Vegfa gene promoter, which contains 3 putative GATA binding sites within $1.3 \mathrm{~kb}$ of the transcription start site (Figure 8A). Transfection of a -240 and -1300 base pair-containing VEGF-A-luciferase promoter reporter into cardiomyocytes showed a 32- and 20-fold upregulation with GATA4 adenoviral infection, respectively (normalized against Ad- $\beta$-gal infection) (Figure 8B). However, transfection of the same VEGF-A-luciferase reporter constructs with an activated calcineurin-expressing adenovirus (Ad- $\Delta \mathrm{CnA}$ ), a known hypertrophic inducer, did not upregulate expression (Figure 8C). EMSA analysis with oligonucleotides corresponding to the 3 putative GATA binding sites showed that sites 1 and 3 were capable of binding GATA4 when compared with a positive control site (Figure 8D; ref. 34 ). The ability of GATA4 to bind the Vegfa gene promoter in vivo was also assessed by chromatin immunoprecipitation (ChIP) from GATA4 DTG hearts, using primers against the promoter region that contained GATA site 1 or the eighth exon as a control (Figure 8E). The data show a significant enrichment of GATA4 binding within the promoter region of the endogenous Vegfa gene but not within the eighth exon, as identified with GATA4-specific antibody and compared with a nonspecific IgG (Figure 8F). Finally, we also mutated the 2 identified GATA binding sites within the -1300 VEGF-A luciferase reporter (Figure 8G), which significantly reduced the ability of GATA4 overexpression to induce this promoter construct in cultured cardiomyocytes (Figure 8H). Collectively these results suggest that GATA4 can directly bind the Vegfa gene promoter and regulate its activity in cardiomyocytes.

GATA4 regulates angiogenesis outside the heart. The myocardium is a highly metabolic tissue, and as such, capillary densi-

\section{Figure 6}

Cardiomyocyte GATA4 regulates capillary-like tube formation in endothelial cells. (A) Representative pictures of HUVEC tube formation when cocultured with Ad- $\beta$-gal, Ad-GATA4, or Ad-GATA4-engrailed infected primary cardiomyocytes on Matrigel. (B) Quantification of relative tube formation of a representative experiment $(n=4$ per condition) in HUVECs cocultured with cardiomyocytes described in $\mathbf{A}$, and the experiment was repeated twice with similar results. ${ }^{*} P<0.001$ versus coculture with Ad- $\beta$-gal and Ad-GATA4-engrailed infected cardiomyocytes; \# $P<0.05$ versus coculture with $\mathrm{Ad}-\beta$-gal-infected cardiomyocytes. (C) Endothelial cell proliferation (assessed by BrdU incorporation with a spectrophotometric absorbance assay) when cocultured with cardiomyocytes infected with Ad- $\beta$-gal, Ad-GATA4, or Ad-GATA4-engrailed infected primary cardiomyocytes. Data from a representative experiment is shown ( $n=7$ per condition). Experiment was repeated twice with similar results. ${ }^{*} P<0.05$ versus coculture with $\mathrm{Ad}-\beta$-gal and Ad-GATA4-engrailed infected cardiomyocytes. Original magnification, $\times 40$. ties cannot be altered beyond a certain point without grave consequences. However, skeletal muscle appears to show more dynamic range in vessel densities following select stimuli $(35,36)$. It was also of interest to look outside the heart to determine whether GATA4regulated angiogenesis was a more global mechanism that applied to other mesoderm-derived tissues. Here we employed a traditional hindlimb ischemia model to assess vessel densities and blood flow after adenoviral infection of GATA4 into skeletal muscle. First, a cohort of mice was injected with Ad-GATA4 or Ad- $\beta$-gal throughout the quadriceps, and the muscle was harvested 7 days later. AdGATA4-injected quadriceps showed robust expression of GATA4
Table 2

GATA4 induces a proangiogenic gene profile in the heart

\begin{tabular}{|c|c|c|c|}
\hline $\begin{array}{l}\text { GenBank } \\
\text { accession no. }\end{array}$ & $\begin{array}{l}\text { Common } \\
\text { gene symbol }\end{array}$ & Product & $\begin{array}{l}\text { Fold change } \\
\text { in DTG }\end{array}$ \\
\hline NM_009311 & Tac1 & Tachykinin 1 & 4.3 \\
\hline NM_011519 & $S d c 1$ & Syndecan 1 & 3 \\
\hline NM_008484 & Lamb3 & Laminin $\beta 3$ & 2.7 \\
\hline NM_172647 & $F 11 r$ & F11 receptor & 2.7 \\
\hline NM_008150 & Gpc4 & Glypican 4 & 2.5 \\
\hline NM_009851 & CD44 & CD44 antigen & 1.9 \\
\hline NM_026993 & Ddah1 & $\begin{array}{c}\text { Dimethylarginine } \\
\text { dimethylaminohydrolase } 1\end{array}$ & 1.9 \\
\hline NM_009642 & AT1R & Angiotensin II, type 1 receptor & 1.8 \\
\hline NM_013655 & Cxc/12 & Stromal cell-derived factor 1 & 1.7 \\
\hline NM_010415 & Dtr & $\begin{array}{l}\text { Heparin-binding EGF-like } \\
\text { growth factor }\end{array}$ & 1.7 \\
\hline NM_009842 & CD151 & CD151 antigen & 1.6 \\
\hline NM_008968 & Ptgis & Prostacyclin synthase & 1.6 \\
\hline NM_011923 & Angpt/2 & Angiopoietin-like 2 & 1.5 \\
\hline NM_011576 & Tfpi & $\begin{array}{l}\text { Tissue factor } \\
\text { pathway inhibitor }\end{array}$ & 0.6 \\
\hline
\end{tabular}

Total RNA was isolated from hearts of tTA and GATA4/tTA DTG mice 2 months after removal of DOX at 1 month of age ( $n=2$ each). Gene expression analysis was carried out with the Mouse Genome 4302.0 Array chip (Affymetrix). All listed genes were significantly changed in expression $(P<0.05)$ in DTG mice. 

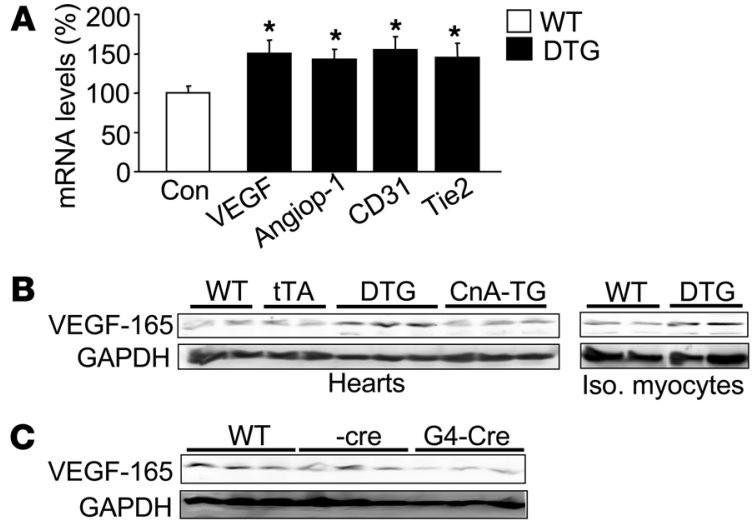

D

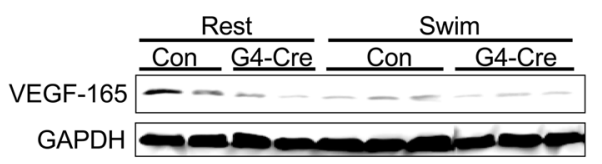

$\mathbf{E}$

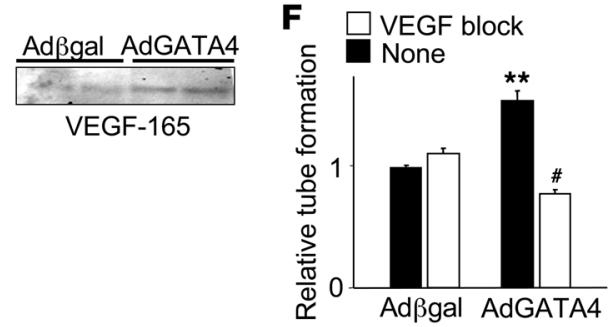

protein by western blotting (Figure 9A). More importantly, AdGATA4-injected skeletal muscle showed a dramatic increase in the density of capillaries, when quantified as capillaries per myofiber or capillaries per microscopic field (Figure 9, B-D). It should be noted that while GATA4 protein is normally not detectable in skeletal muscle (Figure 9A), ectopic expression here simply served as a "proof of concept" for induction of the angiogenic program in another mesoderm-derived tissue. These results suggested that Ad-GATA4-dependent angiogenesis might benefit blood flow in the hindlimb, similar to what was observed in the myocardium. To assess this hypothesis, a second cohort of mice was injected with Ad-GATA4 or Ad- $\beta$-gal (injection into the quadriceps muscle of the right hindlimb) at day 1 , followed by ablation of the femoral artery on day 5 and laser Doppler analysis of blood flow on days 5, 6, and 8. (Figure 9E). While the inhibition of blood flow was equivalent between Ad-GATA4- and Ad- $\beta$-gal-injected legs on day 5 (onset of ischemia), by days 6 and 8 flow was significantly better in Ad-GATA4-treated mice (Figure 9, F and $\mathrm{G}$ ). GATA4 protein overexpression was confirmed in the ischemic hindlimb of the Ad-GATA4-treated mice, which also correlated with induction of endogenous VEGF-165, even though expression is relatively low in skeletal muscle (Figure 9H). Ad-GATA4-treated ischemic hindlimb skeletal muscle after 7 days also showed a significant increase in capillary density, consistent with the observed improvement in flow (Figure 9I). Thus GATA4 overexpression in skeletal muscle induced the angiogenic program and enhanced blood flow, suggesting that GATA4 has inherent angiogenic potential.

\section{Discussion}

Here we identify a novel function for the transcription factor GATA4 as a regulator of angiogenesis in the heart. Our first indi-

\section{Figure 7}

GATA4 induces proangiogenic genes in cardiomyocytes. (A) RNase protection assay of angiogenesis-related genes in GATA4 DTG and control mice (WT and tTA) at 3 months of age (off Dox for 2 months) ( $n$ $=9$ controls and DTG mice). RNA expression in control mice was normalized to $100 \%$. ${ }^{*} P \leq 0.01$ versus control. (B) Western blot analysis of VEGF-165 expression from whole heart (left) and isolated myocytes (right) in WT, tTA, GATA4 DTG, and activated CnA Tg mice. (C) Western blot analysis of VEGF-165 expression from whole heart in WT, $\beta$-Cre, and Gata4 $\beta$-Cre mice. (D) Western blot analysis of VEGF-165 expression from whole heart in control (WT and $\beta$-Cre) and Gata4 $\beta$-Cre mice after 3 weeks of swimming or normal caged activity (rest). GAPDH was used as a loading control throughout. (E) Western blot of VEGF-165 expression in the supernatant of primary cardiomyocytes infected with either Ad- $\beta$-gal or Ad-GATA4 for 48 hours. (F) Quantification of relative tube formation of 1 representative experiment ( $n=3$ per condition) in HUVECs cocultured with cardiomyocytes infected with either Ad$\beta$-gal or Ad-GATA4 in the presence or absence of the VEGF receptor inhibitor CBO-P11 $(12 \mu \mathrm{M})$. The experiment was repeated with similar results. ${ }^{* *} P<0.001$ versus coculture with $A d-\beta$-gal without CBO-P11; ${ }^{\#} P<0.001$ versus coculture with Ad-GATA4 without CBO-P11.

cation that GATA4 might be a proangiogenic factor came from studies in GATA4-overexpressing transgenic mice. While overexpression approaches are sometimes criticized as being unphysiologic, our inducible strategy bypassed any developmental effect that may have occurred in embryos or neonates, and even in adulthood expression was only 4.6- or 2.5-fold above endogenous GATA4 levels. Indeed, this relatively mild level of overexpression in the adult heart likely models the endogenous increase in GATA4 activity that normally occurs following increased hemodynamic load (Figure 4A; refs. 23, 24).

Microvessel expansion has been reported during pressure overload myocardial hypertrophy in multiple species, including humans $(9,12-14)$, suggesting the possibility that GATA4 overexpression had a primary effect on the hypertrophic response that merely impacted vascular density as a secondary consequence. However, we believe this to be unlikely, since line 21.2 GATA4 transgenic mice (low expressing) showed no cardiac hypertrophy well past 7 months of age, yet they maintained greater capillary density and had enhanced coronary flow. Another interesting feature of the GATA4-driven angiogenic response was its maintenance over long periods of time (at least 6 months), in contrast to cardiomyocyte Akt activation in the heart, which only induced a transient angiogenic response (10). Likewise, pressure overload stimulation also induces a transient increase in capillary densities with TAC stimulation (9, 12-14), but long-term hypertrophy, especially in adult humans, has been associated with a decrease in capillary density and cardiac decompensation, myocyte death, and fibrosis $(7,14)$.

Capillary density in the heart is also tightly coupled to cardiomyocyte growth during development $(14,37-39)$. This program is likely recapitulated in the adult heart that is subjected to injury or physiologic stimulation, given that the hypertrophied myocardium also increases vessel growth to ensure adequate perfusion as the myocyte compartment expands, and inhibition of this "matching" effect in microvessels during hypertrophy was recently shown to promote decompensation (9-11). In GATA4-transgenic hearts this increase in capillary density and microvessels resulted in a greater coronary flow reserve and enhanced contractility $(+\mathrm{d} P / \mathrm{d} t)$ upon vasodilation in an isolated working heart preparation. This phenomenon is of significant functional importance, since increased myocardial 
A

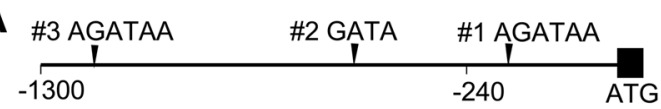

B

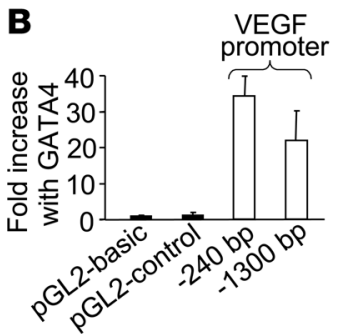

C

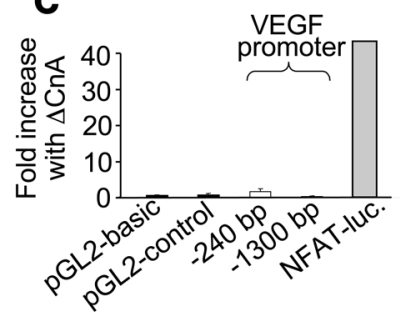

D
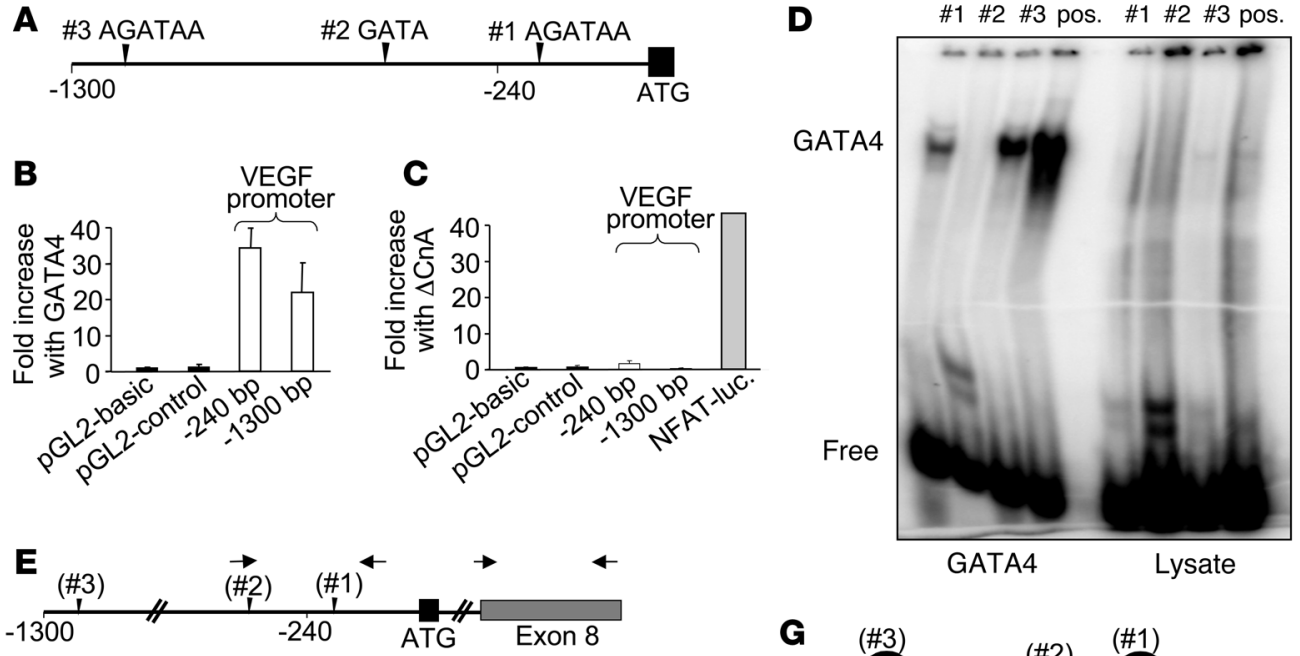

F

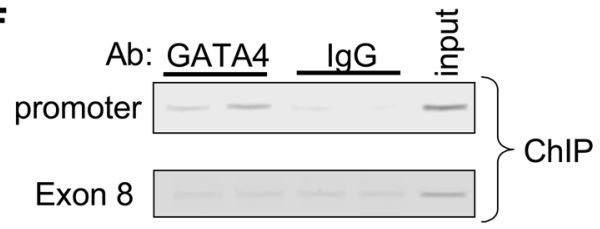

G

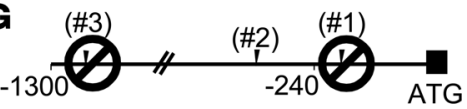

H

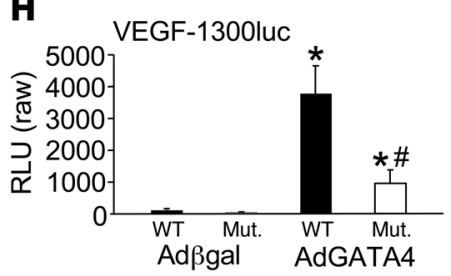

Figure 8

GATA4 directly regulates the Vegfa gene promoter. (A) Schematic representation of the mouse VEGF promoter showing 3 putative GATA binding sites. The number of base pairs upstream of the transcription start site are denoted by -240 and -1300 . (B) VEGF promoter activity shown as fold increase with Ad-GATA4 infection with $A d-\beta$-gal infection set to 1. $p G L 2$-basic is the backbone luciferase reporter vector with the VEGF promoter, while pGL2-control contains the SV40 promoter and is not induced by Ad-GATA4 infection. Results from a representative experiment are shown ( $n=3$ per promoter construct). The experiment was repeated twice with similar results. (C) Results from a similar experiment to that shown in $B$, except that $\mathrm{Ad}-\Delta \mathrm{CnA}$ (activated calcineurin) infection was used instead of Ad-GATA4, and a NFAT-luciferase reporter control adenovirus was used to show the effectiveness of Ad- $\Delta$ CnA. (D) EMSA to detect GATA4 binding to the 3 putative GATA binding sites in the Vegfa gene promoter. Oligonucleotides were incubated with unprogrammed or GATA4-programmed reticulocyte lysate. (E) Schematic of a portion of the Vegfa gene locus showing the position of the 2 primer pairs used for ChIP (arrows). (F) ChIP from GATA4 DTG hearts with GATA4 antibody or a nonspecific IgG to the promoter region or exon 8. (G) Schematic of the Vegfa gene promoter with sites 1 and 3 mutated. $(\mathbf{H})$ Relative luciferase activity from cardiomyocytes transfected with the WT VEGF-1300 luciferase reporter or an identical reporter containing mutations in GATA sites 1 and 3. Myocytes were infected 24 hours prior with Ad- $\beta$-gal or Ad-GATA4. ${ }^{*} P<0.01$ versus WT Ad- $\beta$-gal; $\# P<0.01$ versus WT Ad-GATA4.

oxygen demand, which is present during increased hemodynamic load or exercise, is mainly satisfied by vasodilation and increased perfusion rather than increased oxygen extraction from the blood (40). These data imply that GATA4 serves a compensatory and protective role in the heart by inducing the angiogenic program. These results also suggest that activation of GATA4 in the hypertrophic or pressure-loaded heart might serve as an important mechanism for reemploying the developmental angiogenic response in the heart. Indeed, heart-specific Gata4-deleted mice failed to show enhanced capillary densities following acute pressure overload. More importantly, reinduction of cardiac vascular density in Gata4-deleted mice using VEGF and angiopoietin-1 adenoviral gene transfer partially rescued ventricular dysfunction following 1 week of pressure overload stimulation. Our results strongly suggest that loss tion compared with Ad-GATA4
(data not shown). These observations collectively suggest that GATA4 and GATA6 proteins function in the regulation of angiogenesis/vasculogenesis, although GATA4 may play a more central role, since it directly responds to diverse pathologic and adaptive stimuli and is phosphorylated by multiple kinases as a means of increasing its activity.

Angiogenesis in the adult heart is regulated by 2 potentially distinct mechanisms (with some partial overlap). The first mechanism is the well-known hypoxia-induced response through HIF-1 $\alpha$, which can directly regulate endothelial cell activity and increase capillaries (19). Indeed, in the human myocardium, HIF-1 $\alpha$ activation was only detected in areas that were directly ischemic (44), although a second mechanism was suggested by the observation that HIF-1 $\alpha$ was activated by mechanical overload in mice 


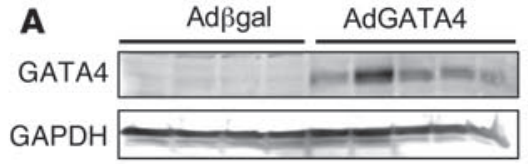

D

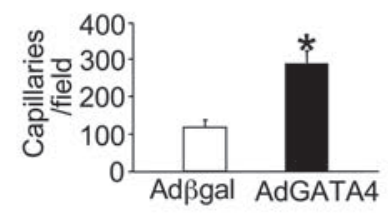

$\mathbf{E}$
B

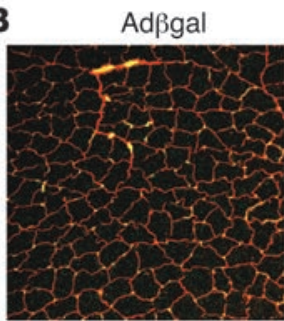

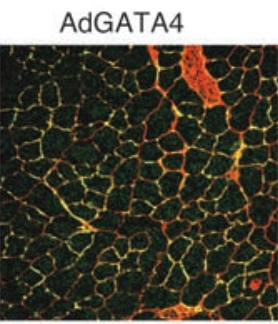

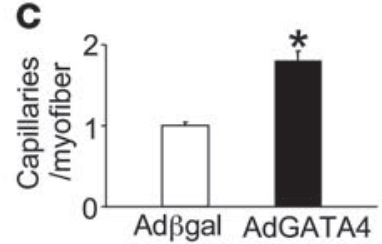

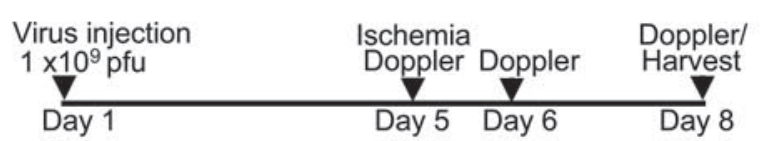

$\mathbf{F}$
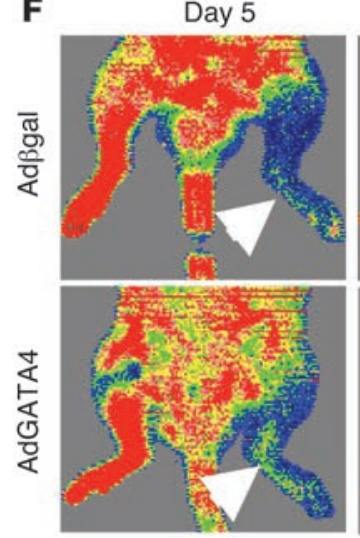

Day 6
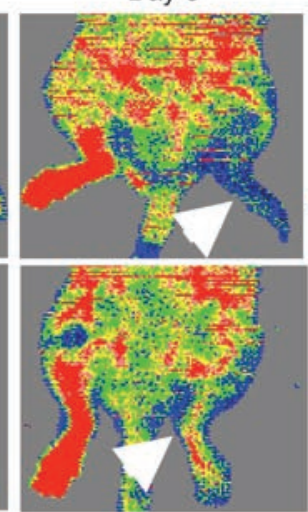

Day 8

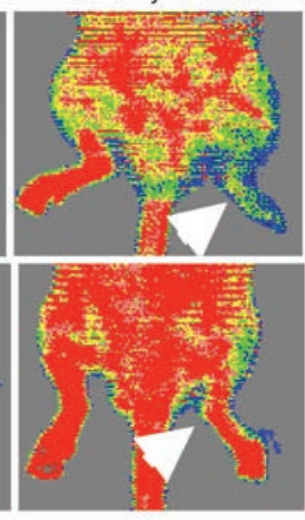

G
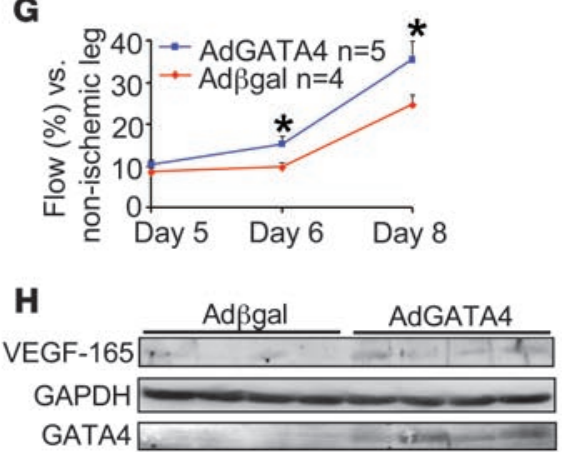

I

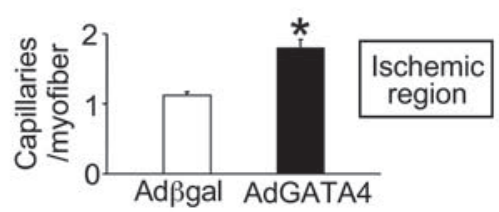

Figure 9

Ad-GATA4 infection induces angiogenesis in quadriceps muscle. (A) Western blot analysis of GATA4 expression in mice 7 days after they were injected with either Ad- $\beta$-gal or Ad-GATA4 throughout their right quadriceps muscle ( $n=4$ Ad- $\beta$-gal mice and $n=5$ Ad-GATA4 mice). (B) Representative images of CD31 staining (green) in quadriceps muscle of mice 7 days after Ad- $\beta$-gal or Ad-GATA4 injection. Counterstaining with WGA was performed (red). (C and D) Quantification of capillaries per field and capillaries per myofiber in mice 7 days after Ad- $\beta$-gal or Ad-GATA4 injection. (E) Experimental time line and procedures used to assess the effect of Ad-GATA4 versus Ad- $\beta$-gal infection in quadriceps muscle on limb perfusion after femoral artery ligation (ischemia) in the right leg. (F) Representative Doppler images of 1 mouse injected with Ad- $\beta$-gal or Ad-GATA4 immediately (day 5), 1 day (day 6), and 3 days (day 8) after femoral artery ligation. Red color indicates greater flow; blue indicates less flow. (G) Quantification of Doppler results from ischemic right leg versus nonischemic left leg in mice injected with Ad- $\beta$-gal (red line) or Ad-GATA4 (blue line). * $P<0.05$ versus Ad- $\beta$-gal-injected mice. (H) Western blot for GATA4 and VEGF-165 in from the ischemic hindlimb muscle 8 days after Ad- $\beta$-gal or Ad-GATA4 infection. (I) Capillaries per myofiber in mice 8 days after Ad- $\beta$-gal or Ad-GATA4 injection from the ischemic hindlimb musculature analyzed in $\mathbf{F}-\mathbf{H}$. Original magnification, $\times 400$.

(45). The second mechanism is associated with a general stress response to the heart following acute hemodynamic overload or exercise- or pregnancy-induced hypertrophy $(9,12-14,35,46)$. Such a response would allow the myocardium to coordinate an increase in microvessels in synchrony with an ensuing hypertrophy response, but without the induction of tissue ischemia. Moreover, this load-induced angiogenic response would protect the heart from developing ischemia in the first place, possibly delaying decompensation. Here we identify GATA4 as a critical regulator of this load-induced angiogenic response in the heart, although we do not address its interplay with the hypoxia response or potential coordination with HIF- $1 \alpha$.

The angiogenic response mediated by GATA4 appears to initiate an entire program of angiogenic cytokines and growth factors, as revealed by Affymetrix array screening. For example, GATA4 DTG hearts showed upregulation of mRNAs for syndecan-1 (47), tachykinin-1 (48), laminin $\beta 3$ (49), and more (Table 2). Moreover, tissue factor pathway inhibitor (tfpi), a known inhibitor of angiogenesis, was found to be downregulated $(50,51)$. A more rigorous quantitative analysis of angiogenic factors by RNase protection assay also revealed significant increases in VEGF and angiopoietin-1 (which were not changed enough to be detected on the Affymetrix array). We believe that induction of this program may be partially direct, since the Vegfa gene was a binding target for GATA4 and given the reciprocal regulation of angiogenesis that occurred in GATA4 gain- and loss-of-function approaches in culture and in the adult mouse heart. Importantly, pharmacological blockade of the VEGF receptor 2 by CBO-P11 completely inhibited GATA4-induced cap- 
illary formation in vitro, indicating that induction of VEGF-A is an essential part of the GATA4-induced angiogenic gene program. Therefore, GATA4 can directly regulate VEGF-A expression and the angiogenic program in the heart through a hypoxia-independent mechanism. These results also highlight a novel mechanism whereby GATA4 promotes cardiac compensation following pathologic stimulation, suggesting a possible novel therapeutic approach to inducing the angiogenic program by GATA4 gene therapy or by other means of increasing its activity.

\section{Methods}

Cardiomyocyte-specific inducible GATA4 transgenic mice and animal procedures. A tetracycline-responsive binary $\alpha$-MHC transgene system (31) was used to allow temporally regulated expression of GATA4 in cardiomyocytes. The mouse GATA4 cDNA was cloned into the SalI-HindIII site of the chimeric tetracycline/Dox-responsive $\alpha$-MHC promoter (responder mice). The construct was linearized and injected into $\mathrm{FVB} / \mathrm{N}$ pronuclei, and founder lines were crossed with transgenic mice expressing the tTA under the control of the constitutive $\alpha$-MHC promoter (FVB/N background). Dox was provided in the food with a special diet formulated by Purina $(625 \mathrm{mg} / \mathrm{kg}$ food) for the first 4 weeks of age to inhibit GATA 4 expression but removed thereafter, resulting in expression of GATA4. Mice expressing the activated calcineurin cDNA (CnA Tg mice; see Figure 2, C and E, and Figure 7B) in the heart and Gata4 ${ }^{\beta}$-Cre gene-targeted animals were both described previously $(29,52)$. TAC was performed as previously described (53). Swimming exercise was conducted for 3 weeks as described previously (29, 53). Animal experiments were approved by the Animal Care and Use Committee of Cincinnati Children's Hospital Medical Center.

Echocardiography and working heart preparation. Mice were anesthetized with $2 \%$ isoflurane, and hearts were visualized using a Hewlett Packard Sonos 5500 instrument and a 15-MHz transducer as described previously in detail (54). An ex vivo isolated working heart preparation was used to measure coronary flow as previously described (55). In brief, the heart was quickly removed from the thoracic cavity, and the aorta was cannulated with a 20-gauge stainless steel cannula, leaving the aortic valve and the coronary artery ostia intact. Another cannula was tied into the pulmonary vein opening of the left atrium. A left ventricular catheter was placed for pressure measurements $(\mathrm{d} P / \mathrm{d} t)$. Anterograde perfusion with $37.7^{\circ} \mathrm{C}$ Krebs-Henseleit solution through the left atrial cannula was adjusted to maintain flow (venous return) at $5 \mathrm{ml} / \mathrm{min}$, and vascular resistance was adjusted to maintain aortic pressure at $50 \mathrm{mmHg}$. The heart was paced at 408 beats $/ \mathrm{min}$. After an adjustment period, the coronary effluent was collected for 5 minutes and weighed, and the coronary flow was calculated in $\mathrm{ml} / \mathrm{min}$. After baseline measurements, nitroprusside (final concentration, $2 \mu \mathrm{g} / \mathrm{min}$ ) was infused into the perfusate, and coronary flow was measured for 5 minutes as described above.

Hindlimb ischemia model and treatment with recombinant adenovirus. Ad- $\beta$-gal or Ad-GATA4 was injected into the quadriceps muscle of the right limb of 8- to 12 -week-old FVB/N mice at a dosage of $1 \times 10^{9} \mathrm{pfu}$ in $150 \mu \mathrm{l}$ of PBS. Four days later, mice were anesthetized with $2 \%$ isoflurane, and the femoral artery of the right limb was isolated and the proximal portion of the artery obliterated using an electrical coagulator. The ratio of ischemic (right) to non-ischemic (left) limb blood flow was measured using a laser Doppler blood flow meter (Laser Doppler Perfusion Imager System, moorLDI2-IR; Moor instruments) immediately after surgery and on days 1 and 3 following surgery. Before scanning was initiated, mice were placed on a heating pad at $37^{\circ} \mathrm{C}$ for 10 minutes. In a second cohort of mice, no ischemia was induced, and 7 days after adenovirus injection the right quadriceps muscle was removed and used for Western blot analysis of GATA4 expression and histological analysis of vessel formation as well. The right quadriceps mus- cle was also removed and used for Western blot and histological analysis in mice with ischemia 8 days after adenovirus injection.

Cardiac adenoviral delivery. Ad-ang-1 and Ad-VEGF were combined and delivered at $2 \times 10^{9} \mathrm{pfu}$ per heart. Dosage of Ad- $\beta$-gal in control experiments was the same as that used in the hindlimb ischemia experiments. Adenovirus was injected with $0.5 \mu \mathrm{g}$ nitroprusside to induce leakiness in capillaries in $0.9 \%$ sodium chloride solution (total volume, $100 \mu \mathrm{l} /$ injection) as a bolus into the left ventricular cavity 10 seconds after cross-clamping the aorta, which was maintained for an additional 30 seconds after vector delivery, as described previously (56). TAC surgery was then performed immediately after the vector delivery during the same surgical intervention.

Western blotting. Western blot analysis was performed using the following antibodies: GATA4, HIF-1 $\alpha$ (Santa Cruz Biotechnology Inc.), LDH, phospho-105-GATA4, VEGF-A mouse (Abcam), and VEGF-A rat (R\&D Systems). Adult cardiomyocytes were isolated as previously described (53). The cell culture supernatant from Ad-GATA4-infected neonatal myocytes was concentrated about 10-fold with Centricon YM-3 columns (Invitrogen) for analysis of VEGF-A expression.

Histological analysis. Paraffin-embedded hearts were used for histological sections for H\&E and Masson's Trichrome staining, the latter of which shows fibrosis in blue. Cryosections were stained with TRITC-conjugated wheat germ agglutinin (Sigma-Aldrich) to outline cardiomyocytes, and immunostaining with CD31 (Chemicon) was used to visualize capillaries and endothelial cells. Conductance vessels were identified by a diameter greater than or equal to $20 \mu \mathrm{m}$ after immunostaining for smooth muscle $\alpha$-actin (Sigma-Aldrich). GATA4 immunostaining was also performed from cryosections. To determine the level of myocardial hypoxia, an EF5-labeling protocol was applied in mice (32). EF5 is a pentafluorinated derivative of etanidazole, which binds cellular macromolecules in hypoxic cells. The total dose of EF5 solution (10 mM dissolved in $0.9 \%$ saline) was given at $4 \%$ of weight by intravenous (50\%) and intraperitoneal (50\%) injection. The hearts were removed 4 hours after EF5 injection and subjected to cryosectioning and immunostaining with Cy3-labeled ELK3-51 antibody against cellular bound EF5 (32). As a positive control, mice were treated with EF5 three hours after ligation of the left coronary artery (57), and as a negative control, uninjected mice were stained with the ELK3-51 antibody. The EF5 and the ELK3-51-Cy3 antibody were generously provided by Cameron J. Koch (University of Pennsylvania School of Medicine, Philadelphia, Pennsylvania, USA). The anti- $\beta$-gal polyclonal antibody was purchased from Chemicon.

$R$ Nase protection assay, microarray analysis, ChIP, and site-directed mutagenesis. Total RNA was isolated from mouse hearts using TRIZOL (Invitrogen). The Multi-Probe RNase Protection Assay system with the mouse Angio Template Set1 (BD Biosciences) was used according to the manufacturer's instructions. For gene expression profiling, the Mouse Genome 4302.0 Array chip (Affymetrix) was used. Total RNA was extracted from 12-weekold tTA control mice $(n=2)$ and GATA4 DTG mice $(n=2)$. Biotin-labeled target cRNA was prepared from T7-transcribed cDNA made from $10 \mu \mathrm{g}$ of total RNA and hybridized for expression analysis using antibody-based fluorescence signal amplification. Genes with significant expression differences between 2 tTA and 2 GATA4 DTG samples were selected from RMA-normalized GeneChip MOE 430 2.0 CEL files based on the assumption of equally distributed upregulated and downregulated genes using Student's $t$ test with a $P$ value cutoff of 0.05 and an average fold change greater than 1.5 (29). ChIP assays were performed as described previously (58) and according to manufacture's directions (ChIP-Assay Kit; Upstate). GATA4 antibody (C-20; Santa Cruz Biotechnology Inc.) and normal goat IgG (Santa Cruz) were used for IP. PCR for Vegfa promoter was performed with the following primers: forward, 5'-CTATCCTCATACGTCCCTGC-3'; reverse, $5^{\prime}$-AGGCCACTACCGCGAAATG-3'. PCR for the Vegfa exon 8 was performed with the following primers: forward, $5^{\prime}$-TCCTCCTCCCAACA- 
CAAGTC-3'; reverse, 5'-CTCTTCGAGGAGCACTTTGG-3'. The quickchange multi site-directed mutagenesis kit from Stratagene was used to mutate the GATA sites (to GAGA) in the -1,300-bp VEGF promoter luciferase reporter vector.

EMSA. EMSA was performed as previously described (34). In brief, double-stranded oligonucleotides corresponding to the 3 putative GATA binding sites in the proximal $1.3 \mathrm{~kb}$ of the mouse VEGF-A promoter were generated (the sense sequence is shown; bold indicates GATA core binding sequence within the oligonucleotides): site 1, 5'-GGTGCACAGATAATTTTGAG-3'; site 2, 5'-GTTGTCCCTATCCTCATACG-3'; site 3, 5'-AGGGCTCAGATAAGCATACT-3'; positive control from the mouse calcineurin A $\beta$ promoter, $5^{\prime}$-TGTACAAAGATAAGGAACTA-3' (34). The sense strand of the oligonucleotides was labeled with $\left[\gamma^{-32} \mathrm{P}\right]$ ATP with T4 polynucleotide kinase (New England Biolabs) before annealing to the other strand. Full-length GATA4 protein was generated using a coupled transcription-translation system (Promega). The annealed oligonucleotides were incubated with GATA4 reticulocyte lysate, and protein-DNA complexes were subsequently separated by nondenaturing gel electrophoresis. Unprogrammed reticulocyte lysate was used as a negative control for each of the binding sites.

Cell culture. The isolation and culture of primary cardiomyocytes from 1- to 2-day-old rat neonates has been described in detail before (34). Cardiomyocytes were infected at an MOI of 25 with Ad-GATA4 or Ad- $\beta$-gal, or an MOI of 100 for Ad-GATA4-engrailed cardiomyocytes. The procedure for infection was described previously (28). HUVECs were purchased from PromoCell and maintained in endothelial growth media according to the supplier's instructions. HUVECs were used for experiments until passage 3 . IGF-1 was recombinant mouse from R\&D systems.

In vitro angiogenesis assay. Formation of capillary-like structures was assessed on growth factor-reduced Matrigel (BD Biosciences; $200 \mu \mathrm{l} /$ well) in 24-well plates. For this procedure, cardiomyocytes were plated overnight on Matrigel (150,000/well) in medium 199 containing 10\% FBS 24 hours after adenoviral infection. The next day, HUVECs $(75,000 /$ well) were plated on top of the cultured cardiomyocytes in endothelial growth media for 1 hour and then switched to serum-free medium 199. After 24 hours, tube formation (total tube length/high-powered field) was quantified using ImageJ software (NIH).

DNA synthesis assay. DNA synthesis was measured by performing a BrdU incorporation assay with a commercially available kit (Roche). Adenoviral- infected cardiomyocytes were plated on gelatinized 96-well plates in medium 199 containing 10\% FBS overnight (5,000 cells/well). The next day, HUVECs were plated on top of the cardiomyocytes in endothelial cell growth media $(5,000$ cells/well) for 8 hours, then switched to serum-free medium 199 overnight. The next morning, BrdU was added to the media for 24 hours, after which BrdU incorporation was determined by ELISA using anti-BrdU antibody.

Luciferase assay. Primary cardiac myocytes were transiently transfected with $2 \mu \mathrm{g} /$ well of 2 different VEGF promoter constructs (-240 bp and $-1300 \mathrm{bp}$ ) in pGL-2 luciferase as well as with 2 different control constructs (pGL-2basic and pGL-2control; Promega), using FuGENE 6 reagent (Roche Molecular Biochemicals). The cells were harvested 24 hours after transfection for luciferase activity determination, as described previously (34). Cardiomyocytes were infected with Ad-GATA4, Ad- $\Delta \mathrm{CNA}$, or Ad- $\beta$-gal 24 hours prior to transfection of the promoter constructs. The VEGF-A promoter constructs were kindly provided by Patricia A. D'Amore (Schepens Eye Research Institute, Boston, Massachusetts, USA).

Statistics. All values are presented as mean \pm SEM. Differences between 2 groups were analyzed by unpaired Student's $t$ test. Differences between 3 or more groups were analyzed by 1-way ANOVA followed by Student-Newman-Keuls post-hoc test. A 2-tailed $P$ value of less than 0.05 was considered to indicate statistical significance.

\section{Acknowledgments}

This work was supported by NIH grants (to J.D. Molkentin). J. Heineke was supported by a Post-Doctoral Fellowship from the Deutsche Forschungsgemeinschaft. J.D. Molkentin is an Established Investigator of the American Heart Association. This work was also supported by an international collaborative research grant in cardiovascular disease from the Leducq Fondation.

Received for publication May 2, 2007, and accepted in revised form September 12, 2007.

Address correspondence to: Jeffery D. Molkentin, Cincinnati Children's Hospital Medical Center, Molecular Cardiovascular Biology, 3333 Burnet Ave. MLC 7020, Cincinnati, Ohio 45229, USA. Phone: (513) 636-3557; Fax (513)-636-5958; E-mail: jeff.molkentin@ cchmc.org.
1. Murray, C.J., and Lopez, A.D. 1997. Alternative projections of mortality and disability by cause 1990-2020: Global Burden of Disease Study. Lancet. 349:1498-1504.

2. Pearson, T.A. 1999. Cardiovascular disease in developing countries: myths, realities, and opportunities. Cardiovasc. Drugs Ther. 13:95-104.

3. Kannel, W.B. 1998. Overview of atherosclerosis. Clin. Ther. 20(Suppl. B):B2-B17.

4. Rosamond, W., et al. 2007. Heart disease and stroke statistics - 2007 update: a report from the American Heart Association Statistics Committee and Stroke Statistics Subcommittee. Circulation. 115:e69-e171.

5. Adams, K.F., Jr. 2001. New epidemiologic perspectives concerning mild-to-moderate heart failure. Am.J. Med. 110(Suppl. 7A):6S-13S.

6. Lloyd-Jones, D.M., et al. 2002. Lifetime risk for developing congestive heart failure: the Framingham Heart Study. Circulation. 106:3068-3072.

7. Hein, S., et al. 2003. Progression from compensated hypertrophy to failure in the pressure-overloaded human heart: structural deterioration and compensatory mechanisms. Circulation. 107:984-991.

8. Heineke, J., and Molkentin, J.D. 2006. Regulation of cardiac hypertrophy by intracellular signalling pathways. Nat. Rev. Mol. Cell Biol. 7:589-600.
9. Sano, M., et al. 2007. p53-induced inhibition of Hif- 1 causes cardiac dysfunction during pressure overload. Nature. 446:444-448.

10. Shiojima, I., et al. 2005. Disruption of coordinated cardiac hypertrophy and angiogenesis contributes to the transition to heart failure. J. Clin. Invest. 115:2108-2118.

11. Izumiya, Y., et al. 2006. Vascular endothelial growth factor blockade promotes the transition from compensatory cardiac hypertrophy to failure in response to pressure overload. Hypertension. 47:887-893.

12. Flanagan, M.F., Fujii, A.M., Colan, S.D., Flanagan, R.G., and Lock, J.E. 1991. Myocardial angiogenesis and coronary perfusion in left ventricular pressureoverload hypertrophy in the young lamb. Evidence for inhibition with chronic protamine administration. Circ. Res. 68:1458-1470.

13. Hilfiker-Kleiner, D., et al. 2005. Lack of JunD promotes pressure overload-induced apoptosis, hypertrophic growth, and angiogenesis in the heart. Circulation. 112:1470-1477.

14. Rakusan, K., Flanagan, M.F., Geva, T., Southern, J., and Van Praagh, R. 1992. Morphometry of human coronary capillaries during normal growth and the effect of age in left ventricular pressure-overload hypertrophy. Circulation. 86:38-46.

15. Giordano, F.J., et al. 2001. A cardiac myocyte vascular endothelial growth factor paracrine pathway is required to maintain cardiac function. Proc. Natl. Acad. Sci. U. S. A. 98:5780-5785.

16. Hsieh, P.C., Davis, M.E., Lisowski, L.K., and Lee, R.T. 2006. Endothelial-cardiomyocyte interactions in cardiac development and repair. Annu. Rev. Physiol. 68:51-66.

17. Kelly, B.D., et al. 2003. Cell type-specific regulation of angiogenic growth factor gene expression and induction of angiogenesis in nonischemic tissue by a constitutively active form of hypoxia-inducible factor 1. Circ. Res. 93:1074-1081.

18. Suri, C., et al. 1996. Requisite role of angiopoietin1, a ligand for the TIE2 receptor, during embryonic angiogenesis. Cell. 87:1171-1180.

19. Pugh, C.W., and Ratcliffe, P.J. 2003. Regulation of angiogenesis by hypoxia: role of the HIF system. Nat. Med. 9:677-684.

20. Li, J., Hampton, T., Morgan, J.P., and Simons, M. 1997. Stretch-induced VEGF expression in the heart. J. Clin. Invest. 100:18-24.

21. Zheng, W., Seftor, E.A., Meininger, C.J., Hendrix, M.J., and Tomanek, R.J. 2001. Mechanisms of coronary angiogenesis in response to stretch: role 
of VEGF and TGF-beta. Am. J. Physiol. Heart Circ. Physiol. 280:H909-H917.

22. Oka, T., Xu, J., and Molkentin, J.D. 2007. Re-employment of developmental transcription factors in adult heart disease. Semin. Cell Dev. Biol. 18:117-131.

23. Hautala, N., Tenhunen, O., Szokodi, I., and Ruskoaho, H. 2002. Direct left ventricular wall stretch activates GATA4 binding in perfused rat heart: involvement of autocrine/paracrine pathways. Pflugers Arch. 443:362-369.

24. Hautala, N., et al. 2001. Pressure overload increases GATA4 binding activity via endothelin-1. Circulation. 103:730-735.

25. Charron, F., et al. 2001. Tissue-specific GATA factors are transcriptional effectors of the small GTPase RhoA. Genes Dev. 15:2702-2719.

26. Liang, Q., et al. 2001. The transcription factor GATA4 is activated by extracellular signal-regulated kinase 1- and 2-mediated phosphorylation of serine 105 in cardiomyocytes. Mol. Cell. Biol. 21:7460-7469.

27. Morisco, C., et al. 2001. Glycogen synthase kinase 3 beta regulates GATA4 in cardiac myocytes. J. Biol. Chem. 276:28586-28597.

28. Liang, Q., et al. 2001. The transcription factors GATA4 and GATA6 regulate cardiomyocyte hypertrophy in vitro and in vivo. J. Biol. Chem. 276:30245-30253.

29. Oka, T., et al. 2006. Cardiac-specific deletion of Gata4 reveals its requirement for hypertrophy, compensation, and myocyte viability. Circ. Res. 98:837-845.

30. Bisping, E., et al. 2006. Gata4 is required for maintenance of postnatal cardiac function and protection from pressure overload-induced heart failure. Proc. Natl. Acad. Sci. U. S. A. 103:14471-14476

31. Sanbe, A., et al. 2003. Reengineering inducible cardiac-specific transgenesis with an attenuated myosin heavy chain promoter. Circ. Res. 92:609-616.

32. Maity, A., Sall, W., Koch, C.J., Oprysko, P.R., and Evans, S.M. 2001. Low pO2 and beta-estradiol induce VEGF in MCF-7 and MCF-7-5C cells: relationship to in vivo hypoxia. Breast Cancer Res. Treat. 67:51-60.

33. Yancopoulos, G.D., et al. 2000. Vascular-specific growth factors and blood vessel formation. Nature. 407:242-248.

34. Oka, T., Dai, Y.S., and Molkentin, J.D. 2005. Regula- tion of calcineurin through transcriptional induction of the calcineurin A beta promoter in vitro and in vivo. Mol. Cell. Biol. 25:6649-6659.

35. Jacobs, T.B., Bell, R.D., and McClements, J.D. 1984. Exercise, age and the development of the myocardial vasculature. Growth. 48:148-157.

36. Waters, R.E., Rotevatn, S., Li, P., Annex, B.H., and Yan, Z. 2004. Voluntary running induces fiber typespecific angiogenesis in mouse skeletal muscle. Am. J. Physiol. Cell Physiol. 287:C1342-C1348.

37. Smolich, J.J., Walker, A.M., Campbell, G.R., and Adamson, T.M. 1989. Left and right ventricular myocardial morphometry in fetal, neonatal, and adult sheep. Am. J. Physiol. 257:H1-H9.

38. Tomanek, R.J., Searls, J.C., and Lachenbruch, P.A. 1982. Quantitative changes in the capillary bed during developing, peak, and stabilized cardiac hypertrophy in the spontaneously hypertensive rat. Circ. Res. 51:295-304.

39. Tomanek, R.J. 1992. Age as a modulator of coronary capillary angiogenesis. Circulation. 86:320-321.

40. Tune, J.D., Gorman, M.W., and Feigl, E.O. 2004 Matching coronary blood flow to myocardial oxygen consumption. J. Appl. Physiol. 97:404-415.

41. Huang, Y., et al. 2004. Cardiac myocyte-specific HIF-1alpha deletion alters vascularization, energy availability, calcium flux, and contractility in the normoxic heart. FASEB J. 18:1138-1140.

42. Molkentin, J.D. 2000. The zinc finger-containing transcription factors GATA-4, -5 , and -6 . Ubiquitously expressed regulators of tissue-specific gene expression. J. Biol. Chem. 275:38949-38952.

43. Xin, M., et al. 2006. A threshold of GATA4 and GATA6 expression is required for cardiovascular development. Proc. Natl. Acad. Sci. U. S. A. 103:11189-11194.

44. Lee, S.H., et al. 2000. Early expression of angiogenesis factors in acute myocardial ischemia and infarction. N. Engl. J. Med. 342:626-633.

45. Kim, C.H., Cho, Y.S., Chun, Y.S., Park, J.W., and Kim, M.S. 2002. Early expression of myocardial HIF-1alpha in response to mechanical stresses: regulation by stretch-activated channels and the phosphatidylinositol 3-kinase signaling pathway. Circ. Res. 90:E25-E33.

46. Hilfiker-Kleiner, D., et al. 2007. A cathepsin Dcleaved $16 \mathrm{kDa}$ form of prolactin mediates post- partum cardiomyopathy. Cell. 128:589-600.

47. Maeda, T., Desouky, J., and Friedl, A. 2006. Syndecan-1 expression by stromal fibroblasts promotes breast carcinoma growth in vivo and stimulates tumor angiogenesis. Oncogene. 25:1408-1412.

48. Walsh, D.A., and McWilliams, D.F. 2006. Tachykinins and the cardiovascular system. Curr. Drug Targets. 7:1031-1042.

49. Patarroyo, M., Tryggvason, K., and Virtanen, I. 2002. Laminin isoforms in tumor invasion, angiogenesis and metastasis. Semin. Cancer Biol. 12:197-207.

50. Carmeliet, P., et al. 1996. Role of tissue factor in embryonic blood vessel development. Nature. 383:73-75.

51. Mousa, S.A., and Mohamed, S. 2004. Inhibition of endothelial cell tube formation by the low molecular weight heparin, tinzaparin, is mediated by tissue factor pathway inhibitor. Thromb. Haemost. 92:627-633.

52. Molkentin, J.D., et al. 1998. A calcineurin-dependent transcriptional pathway for cardiac hypertrophy. Cell. 93:215-228.

53. Wilkins, B.J., et al. 2004. Calcineurin/NFAT coupling participates in pathological, but not physiological, cardiac hypertrophy. Circ. Res. 94:110-118.

54. Xu, J., et al. 2006. GDF15/MIC-1 functions as a protective and antihypertrophic factor released from the myocardium in association with SMAD protein activation. Circ. Res. 98:342-350.

55. House, S.L., et al. 2003. Cardiac-specific overexpression of fibroblast growth factor- 2 protects against myocardial dysfunction and infarction in a murine model of low-flow ischemia. Circulation. 108:3140-3148

56. Roth, D.M., et al. 2004. Indirect intracoronary delivery of adenovirus encoding adenylyl cyclase increases left ventricular contractile function in mice. Am. J. Physiol. Heart Circ. Physiol. 287:H172-H177.

57. Heineke, J., et al. 2005. Attenuation of cardiac remodeling after myocardial infarction by muscle LIM protein-calcineurin signaling at the sarcomeric Z-disc. Proc. Natl. Acad. Sci. U. S. A. 102:1655-1660.

58. Dai, Y.S., Xu, J., and Molkentin, J.D. 2005. The DnaJ-related factor Mrj interacts with nuclear factor of activated $\mathrm{T}$ cells $\mathrm{c} 3$ and mediates transcriptional repression through class II histone deacetylase recruitment. Mol. Cell. Biol 25:9936-9948. 